This is a self-archived version of an original article. This version may differ from the original in pagination and typographic details.

Author(s): Ghosh, Tuhin; Salo, Mikko; Uhlmann, Gunther

Title: The Calderón problem for the fractional Schrödinger equation

Year: 2020

Version: Published version

Copyright: @ 2020 Mathematical Sciences Publishers

Rights: In Copyright

Rights url: http://rightsstatements.org/page//nC/1.0/?language=en

Please cite the original version:

Ghosh, T., Salo, M., \& UhImann, G. (2020). The Calderón problem for the fractional Schrödinger equation. Analysis and PDE, 13(2), 455-475. https://doi.org/10.2140/apde.2020.13.455 


\section{ANALYSIS \& PDE}

\section{Volume $13 \quad$ No.2 2020}

TUHIN GHOSH, MIKKO SALO AND GUNTHER UHLMANN

\section{THE CALDERÓN PROBLEM FOR}

\section{THE FRACTIONAL SCHRÖDINGER EQUATION}




\title{
THE CALDERÓN PROBLEM FOR THE FRACTIONAL SCHRÖDINGER EQUATION
}

\author{
Tuhin Ghosh, MikKo Salo And Gunther Uhlmann
}

\begin{abstract}
We show global uniqueness in an inverse problem for the fractional Schrödinger equation: an unknown potential in a bounded domain is uniquely determined by exterior measurements of solutions. We also show global uniqueness in the partial data problem where measurements are taken in arbitrary open, possibly disjoint, subsets of the exterior. The results apply in any dimension $\geq 1$ and are based on a strong approximation property of the fractional equation that extends earlier work. This special feature of the nonlocal equation renders the analysis of related inverse problems radically different from the traditional Calderón problem.
\end{abstract}

\section{Introduction}

In this article we consider a nonlocal analogue of the inverse conductivity problem posed in [Calderón 1980]. In the standard Calderón problem, the objective is to determine the electrical conductivity of a medium from voltage and current measurements on its boundary. This problem is the mathematical model of electrical resistivity/impedance tomography in seismic, medical and industrial imaging. It serves as a model case for various inverse problems for elliptic equations, and has a rich mathematical theory with connections to many other questions. We refer to the survey [Uhlmann 2014] for more details.

In mathematical terms, if $\Omega \subset \mathbb{R}^{n}$ is a bounded open set with Lipschitz boundary (the medium of interest), after a standard reduction one often considers the Dirichlet problem for the Schrödinger equation

$$
(-\Delta+q) u=0 \quad \text { in } \Omega,\left.\quad u\right|_{\partial \Omega}=f,
$$

where $q \in L^{\infty}(\Omega)$ and 0 is not a Dirichlet eigenvalue for $-\Delta+q$ in $\Omega$. The boundary measurements are given by the Dirichlet-to-Neumann map (DN map)

$$
\Lambda_{q}: H^{1 / 2}(\partial \Omega) \rightarrow H^{-1 / 2}(\partial \Omega),
$$

defined weakly in terms of the bilinear form for the equation. Here and below, we denote the standard $L^{2}$ based Sobolev spaces by $H^{s}$.

For more regular boundaries and functions $f$, the DN map is given by the normal derivative $\Lambda_{q} f=$ $\left.\partial_{\nu} u\right|_{\partial \Omega}$, where $u$ is the solution with boundary value $f$. The inverse problem is to determine the potential $q$ in $\Omega$ from the knowledge of the DN map $\Lambda_{q}$.

MSC2010: primary 26A33, 35J10, 35R30; secondary 35J70.

Keywords: inverse problem, Calderón problem, fractional Laplacian, approximation property. 
We will consider an inverse problem for a nonlocal analogue of the Schrödinger equation. In fact, our equation will be the fractional Schrödinger equation $\left((-\Delta)^{s}+q\right) u=0$ in $\Omega$, where $0<s<1$. Here the fractional Laplacian is defined by

$$
(-\Delta)^{s} u=\mathscr{F}^{-1}\left\{|\xi|^{2 s} \hat{u}(\xi)\right\}, \quad u \in H^{s}\left(\mathbb{R}^{n}\right),
$$

and $\hat{u}=\mathscr{F} u$ is the Fourier transform of $u$. This operator is nonlocal (it does not preserve the support of $u$ ), and one natural way to set up the Dirichlet problem is to look for solutions $u \in H^{s}\left(\mathbb{R}^{n}\right)$ satisfying

$$
\left((-\Delta)^{s}+q\right) u=0 \quad \text { in } \Omega,\left.\quad u\right|_{\Omega_{e}}=f,
$$

where $f \in H^{s}\left(\Omega_{e}\right)$, and $\Omega_{e}$ is the exterior domain

$$
\Omega_{e}=\mathbb{R}^{n} \backslash \bar{\Omega} .
$$

We recall basic facts about weak solutions in Section 2. In particular, there is a countable set of Dirichlet eigenvalues, and we will assume that $q$ is such that 0 is not an eigenvalue; that is,

$$
\text { if } u \in H^{s}\left(\mathbb{R}^{n}\right) \text { solves }\left((-\Delta)^{s}+q\right) u=0 \text { in } \Omega \text { and }\left.u\right|_{\Omega_{e}}=0 \text {, then } u \equiv 0 \text {. }
$$

This holds, e.g., if $q \geq 0$. Then there is a unique solution $u \in H^{s}\left(\mathbb{R}^{n}\right)$ for any $f \in H^{s}\left(\Omega_{e}\right)$, and one may define an analogue of the DN map,

$$
\Lambda_{q}: H^{s}\left(\Omega_{e}\right) \rightarrow H^{s}\left(\Omega_{e}\right)^{*},
$$

that maps $f$ to a nonlocal analogue of the Neumann boundary value of the solution $u$. (This discussion assumes that $\Omega$ is a bounded Lipschitz domain; see Section 2 for the case of general bounded open sets.)

We will define $\Lambda_{q}$ in Section 2 via the bilinear form associated with the fractional Dirichlet problem, which will be sufficient for the proof of Theorem 1.1. There are other nonlocal Neumann operators that one could use, but by Theorem 1.1 any reasonable measurement operator would be determined by $\Lambda_{q}$; we will verify this directly for the operator $\mathcal{N}_{s}$ in [Dipierro et al. 2017a]. Again, if $\Omega$ has $C^{\infty}$ boundary and $q$ and $f$ are more regular, the DN map is more explicit and is given by

$$
\Lambda_{q}: H^{s+\beta}\left(\Omega_{e}\right) \rightarrow H^{-s+\beta}\left(\Omega_{e}\right), \quad \Lambda_{q} f=\left.(-\Delta)^{s} u\right|_{\Omega_{e}},
$$

where $u$ is the solution of $\left((-\Delta)^{s}+q\right) u=0$ in $\Omega$ with exterior value $f$, and $\max \left\{0, s-\frac{1}{2}\right\}<\beta<\frac{1}{2}$ (such a $\beta$ exists since $0<s<1$ ). Heuristically, given an open set $W \subset \Omega_{e}$, one can interpret $\left.\Lambda_{q} f\right|_{W}$ as measuring the cost required to maintain the exterior value $f$ in $W$. For more details on these facts (which will not be needed for the proof of Theorem 1.1), we refer to the Appendix.

The following theorem is the main result in this article. It solves the fractional Schrödinger inverse problem in any dimension $n \geq 1$, and also the partial data problem with exterior Dirichlet and Neumann measurements in arbitrary open (possibly disjoint) sets $W_{1}, W_{2} \subset \Omega_{e}$.

Theorem 1.1. Let $\Omega \subset \mathbb{R}^{n}, n \geq 1$, be bounded open, let $0<s<1$, and let $q_{1}, q_{2} \in L^{\infty}(\Omega)$ satisfy (1-1). Let also $W_{1}, W_{2} \subset \Omega_{e}$ be open. If the DN maps for the equations $\left((-\Delta)^{s}+q_{j}\right) u=0$ in $\Omega$ satisfy

$$
\left.\Lambda_{q_{1}} f\right|_{W_{2}}=\left.\Lambda_{q_{2}} f\right|_{W_{2}} \quad \text { for any } f \in C_{c}^{\infty}\left(W_{1}\right),
$$

then $q_{1}=q_{2}$ in $\Omega$. 
For the usual Schrödinger equation $(-\Delta+q) u=0$ and the related DN map $\Lambda_{q}$ on the full boundary $\partial \Omega$, the corresponding result is due to [Sylvester and Uhlmann 1987] when $n \geq 3$ and to [Bukhgeim 2008] when $n=2$ for slightly more regular potentials; for the case of $L^{p}$ potentials see [Blåsten et al. 2015] when $n=2$ and [Chanillo 1990; Nachman 1992] when $n \geq 3$. The partial data problem of determining $q$ from the knowledge of $\left.\Lambda_{q} f\right|_{\Gamma}$ for any $f$ supported in $\Gamma$, when $\Gamma$ is an arbitrary open subset of $\partial \Omega$, was solved in [Imanuvilov et al. 2010] when $n=2$ for $q_{j} \in C^{2, \alpha}$. The corresponding result in dimensions $n \geq 3$ is open, but there are several partial results including [Kenig et al. 2007; Isakov 2007; Kenig and Salo 2013]. The case of measurements on disjoint sets is even more difficult, and counterexamples may appear [Imanuvilov et al. 2011; Daudé et al. 2019a; 2019b]. See the surveys [Imanuvilov and Yamamoto 2013; Kenig and Salo 2014] for further references.

The proof of Theorem 1.1 begins by showing that if the two DN maps are equal, then (exactly as in the usual Schrödinger case) one has the integral identity

$$
\int_{\Omega}\left(q_{1}-q_{2}\right) u_{1} u_{2} d x=0
$$

for any $u_{j} \in H^{s}\left(\mathbb{R}^{n}\right)$ that solve $\left((-\Delta)^{s}+q_{j}\right) u_{j}=0$ in $\Omega$ and satisfy $\operatorname{supp}\left(u_{j}\right) \subset \bar{\Omega} \cup \bar{W}_{j}$. For the standard Schrödinger equation, one then typically uses special complex geometrical optics solutions $u_{j}$ to show that the products $\left\{u_{1} u_{2}\right\}$ form a complete set in $L^{1}(\Omega)$. See [Uhlmann 2014] for an overview.

However, solutions of the fractional Schrödinger equation are much less rigid than those of the usual Schrödinger equation. The fractional equation enjoys stronger uniqueness and approximation properties, as demonstrated by the following theorems:

Theorem 1.2. If $0<s<1$, if $u \in H^{-r}\left(\mathbb{R}^{n}\right)$ for some $r \in \mathbb{R}$, and if both $u$ and $(-\Delta)^{s} u$ vanish in some open set, then $u \equiv 0$.

Theorem 1.3. Let $\Omega \subset \mathbb{R}^{n}$ be a bounded open set, and let $\Omega_{1} \subset \mathbb{R}^{n}$ be any open set with $\Omega \subset \Omega_{1}$ and $\Omega_{1} \backslash \bar{\Omega} \neq \varnothing$ :

(a) If $q \in L^{\infty}(\Omega)$ satisfies (1-1), then any $f \in L^{2}(\Omega)$ can be approximated arbitrarily well in $L^{2}(\Omega)$ by functions $\left.u\right|_{\Omega}$ where $u \in H^{s}\left(\mathbb{R}^{n}\right)$ satisfy

$$
\left((-\Delta)^{s}+q\right) u=0 \quad \text { in } \Omega, \quad \operatorname{supp}(u) \subset \bar{\Omega}_{1} .
$$

(b) If $\Omega$ has $C^{\infty}$ boundary, and if $q \in C_{c}^{\infty}(\Omega)$ satisfies (1-1), then any $f \in C^{\infty}(\bar{\Omega})$ can be approximated arbitrarily well in $C^{\infty}(\bar{\Omega})$ by functions $\left.d(x)^{-s} u\right|_{\Omega}$ where $u \in H^{s}\left(\mathbb{R}^{n}\right)$ satisfy

$$
\left((-\Delta)^{s}+q\right) u=0 \quad \text { in } \Omega, \quad \operatorname{supp}(u) \subset \bar{\Omega}_{1} .
$$

(Here $d$ is any function in $C^{\infty}(\bar{\Omega})$ with $d(x)=\operatorname{dist}(x, \partial \Omega)$ near $\Omega$ and $d>0$ in $\Omega$. Also, $v_{j} \rightarrow v$ in $C^{\infty}(\bar{\Omega})$ means that $v_{j} \rightarrow v$ in $C^{k}(\bar{\Omega})$ for all $k \geq 0$.)

Note that both of these properties fail for the usual Laplacian: if $u \in C_{c}^{\infty}\left(\mathbb{R}^{n}\right)$ then both $u$ and $\Delta u$ vanish in a large set but $u$ can be nontrivial, and the set of harmonic functions in $L^{2}(\Omega)$ is a closed subspace of $L^{2}(\Omega)$ which is smaller than $L^{2}(\Omega)$. 
Theorem 1.2 is classical [Riesz 1938] at least with stronger conditions on $u$, and even the strong unique continuation principle holds in this context [Fall and Felli 2014; Rüland 2015; Yu 2017]. For later applications we will give a robust proof using the Carleman estimates from [Rüland 2015] and the Caffarelli-Silvestre extension [2007].

The following version of Theorem 1.3 has been proved in [Dipierro et al. 2017b]; see also [Dipierro et al. 2019]: given $f \in C^{k}\left(\bar{B}_{1}\right)$ and $\varepsilon>0$, there is $u \in H^{s}\left(\mathbb{R}^{n}\right)$ with $(-\Delta)^{s} u=0$ in $B_{1}$ and $\operatorname{supp}(u) \subset \bar{B}_{R}$ for some possibly large $R=R_{\varepsilon, f}>1$, so that

$$
\|u-f\|_{C^{k}\left(\bar{B}_{1}\right)}<\varepsilon .
$$

Theorem 1.3 improves this by reducing the approximation property to the uniqueness property, Theorem 1.2, using a Runge-type argument [Lax 1956; Malgrange 1956] and regularity for fractional Dirichlet problems [Hörmander 1965; Grubb 2015]. In particular, this implies that the result of [Dipierro et al. 2017b] is valid for any fixed $R>1$. The strong approximation property replaces the method of complex geometrical optics in solving the inverse problem for the fractional Schrödinger equation.

The study of fractional and nonlocal operators is currently an active research field and the related literature is substantial. We only mention that operators of this type arise in problems involving anomalous diffusion and random processes with jumps, and they have applications in probability theory, physics, finance, and biology. See [Bucur and Valdinoci 2016; Ros-Oton 2016] for further information and references.

The mathematical study of inverse problems for fractional equations goes back at least to [Cheng et al. 2009]. By now there are a number of results, mostly for time-fractional models and including many numerical works. Here is an example of the rigorous results that are available [Sakamoto and Yamamoto 2011]: in the time-fractional heat equation

$$
\partial_{t}^{\alpha} u-\Delta u=0 \quad \text { in } \Omega \times(0, T),\left.\quad u\right|_{\partial \Omega \times(0, T)}=0,
$$

where $0<\alpha<1$ and $\partial_{t}^{\alpha}$ is the Caputo derivative, $u(0)$ is determined by $u(T)$ in a mildly ill-posed way (for $\alpha=1$ this problem is severely ill-posed). In general, nonlocality may influence the nature of the inverse problem but there are several aspects to be taken into account. We refer to [Jin and Rundell 2015] for a detailed discussion and many further references. We are not aware of any previous rigorous works on multidimensional inverse problems for space-fractional equations.

Finally, we note that Theorem 1.1 is a global uniqueness result in the inverse problem for the fractional Schrödinger equation, both with full and partial data. This provides a starting point for further work on inverse problems for fractional equations and nonlocal models. In fact, after this article was first submitted as a preprint, several works that build upon the ideas introduced here have appeared. These include results for low regularity and stability [Rüland and Salo 2018; 2019a], matrix coefficients [Ghosh et al. 2017], semilinear equations [Lai and Lin 2019], reconstruction and shape detection [Harrach and Lin 2017; Cao et al. 2019; Ghosh et al. 2018], and quantitative Runge approximation [Rüland and Salo 2019b; 2020]. See also the survey [Salo 2017].

This paper is organized as follows. Section 1 is the introduction. In Section 2 we review weak solutions of fractional Dirichlet problems, and give a definition of the DN map. In Sections 3 and 4 we prove 
Theorems 1.2 and 1.3(a). The solution of the inverse problem, Theorem 1.1, is given in Section 5. In Section 6 we invoke the regularity theory for fractional Dirichlet problems in [Grubb 2015] and prove Theorem 1.3(b). Further properties and alternative descriptions of the DN map may be found in the Appendix.

\section{Fractional Laplacian}

In this section we review some basic facts about Dirichlet problems for the fractional Laplacian; see, e.g., [Hoh and Jacob 1996; Felsinger et al. 2015; Grubb 2015; Ros-Oton 2016]. For simplicity, we will assume most functions to be real-valued in this paper.

2A. Sobolev spaces. We first establish the notation for Sobolev-type spaces. We write $H^{s}\left(\mathbb{R}^{n}\right)=$ $W^{s, 2}\left(\mathbb{R}^{n}\right)$ for the standard $L^{2}$ based Sobolev space with norm

$$
\|u\|_{H^{s}\left(\mathbb{R}^{n}\right)}=\left\|\langle D\rangle^{s} u\right\|_{L^{2}\left(\mathbb{R}^{n}\right)},
$$

where $\langle\xi\rangle=\left(1+|\xi|^{2}\right)^{1 / 2}$, and the notation $m(D) u=\mathscr{F}^{-1}\{m(\xi) \hat{u}(\xi)\}$ is used for Fourier multipliers when $m \in C^{\infty}\left(\mathbb{R}^{n}\right)$ is polynomially bounded together with its derivatives. Our notation for the Fourier transform is

$$
\hat{u}(\xi)=\mathscr{F} u(\xi)=\int_{\mathbb{R}^{n}} e^{-i x \cdot \xi} u(x) d x .
$$

If $U \subset \mathbb{R}^{n}$ is an open set (not necessarily bounded), define the spaces (we follow the notation of [McLean 2000])

$$
\begin{aligned}
& H^{s}(U)=\left\{\left.u\right|_{U}: u \in H^{s}\left(\mathbb{R}^{n}\right)\right\}, \\
& \widetilde{H}^{s}(U)=\text { closure of } C_{c}^{\infty}(U) \text { in } H^{s}\left(\mathbb{R}^{n}\right), \\
& H_{0}^{s}(U)=\text { closure of } C_{c}^{\infty}(U) \text { in } H^{s}(U) .
\end{aligned}
$$

We equip $H^{s}(U)$ with the quotient norm $\|u\|_{H^{s}(U)}=\inf \left\{\|w\|_{H^{s}}: w \in H^{s}\left(\mathbb{R}^{n}\right),\left.w\right|_{U}=u\right\}$. Also, if $F \subset \mathbb{R}^{n}$ is a closed set, we define

$$
H_{F}^{s}=H_{F}^{s}\left(\mathbb{R}^{n}\right)=\left\{u \in H^{s}\left(\mathbb{R}^{n}\right): \operatorname{supp}(u) \subset F\right\} .
$$

We say that an open set $U \subset \mathbb{R}^{n}$ is a Lipschitz domain if its boundary $\partial U$ is compact and if locally near each boundary point $U$ can be represented as the set above the graph of a Lipschitz function. Thus $U$ could be a bounded Lipschitz domain, or $U$ could be $\mathbb{R}^{n} \backslash \bar{\Omega}$, where $\Omega$ is a bounded Lipschitz domain. If $U$ is a Lipschitz domain, then (with natural identifications, see [McLean 2000; Triebel 2002])

$$
\begin{gathered}
\widetilde{H}^{s}(U)=H_{\bar{U}}^{s}\left(\mathbb{R}^{n}\right), \quad s \in \mathbb{R}, \\
H_{\bar{U}}^{s}\left(\mathbb{R}^{n}\right)^{*}=H^{-s}(U) \quad \text { and } \quad H^{s}(U)^{*}=H_{\bar{U}}^{-s}\left(\mathbb{R}^{n}\right), \quad s \in \mathbb{R}, \\
H^{s}(U)=H_{\bar{U}}^{s}\left(\mathbb{R}^{n}\right)=H_{0}^{s}(U), \quad-\frac{1}{2}<s<\frac{1}{2} .
\end{gathered}
$$

2B. Fractional Laplacian. Let $s>-n / 2$ and consider the fractional Laplacian in $\mathbb{R}^{n}$,

$$
(-\Delta)^{s} u=\mathscr{F}^{-1}\left\{|\xi|^{2 s} \hat{u}(\xi)\right\}, \quad u \in \mathscr{S},
$$


where $\mathscr{S}$ denotes Schwartz space in $\mathbb{R}^{n}$. If $\psi \in C_{c}^{\infty}\left(\mathbb{R}^{n}\right)$ with $\psi=1$ near 0 , writing $|\xi|^{2 s}=\psi(\xi)|\xi|^{2 s}+$ $(1-\psi(\xi))|\xi|^{2 s}$ and using the assumption $s>-n / 2$ shows that $|\xi|^{2 s}$ is the sum of an $L^{1}$ function and a smooth function whose derivatives grow at most polynomially. Thus $(-\Delta)^{s}$ for $s>-n / 2$ is a continuous map from $\mathscr{S}$ to $L^{\infty}$.

There are many other definitions of the fractional Laplacian [Kwaśnicki 2017]. For instance, if $0<s<1$, it is given by the principal value integral

$$
(-\Delta)^{s} u(x)=c_{n, s} \text { p.v. } \int_{\mathbb{R}^{n}} \frac{u(x)-u(y)}{|x-y|^{n+2 s}} d y .
$$

We next extend $(-\Delta)^{s}$ to act on larger spaces. In particular, if $s \geq 0$, then $(-\Delta)^{s}$ will be well-defined on $H^{r}\left(\mathbb{R}^{n}\right)$ for any $r \in \mathbb{R}$.

Lemma 2.1. If $s \geq 0$, the fractional Laplacian extends as a bounded map

$$
(-\Delta)^{s}: H^{r}\left(\mathbb{R}^{n}\right) \rightarrow H^{r-2 s}\left(\mathbb{R}^{n}\right)
$$

whenever $r \in \mathbb{R}$. If $-n / 2<s<0$, the fractional Laplacian $(-\Delta)^{s}$ is the Riesz potential

$$
(-\Delta)^{s} u=I_{2|s|} u=\frac{c_{n, s}}{|\cdot|^{n-2|s|}} * u
$$

and it extends as a bounded map

Proof. If $u \in \mathscr{S}$, then

$$
(-\Delta)^{s}: L^{p}\left(\mathbb{R}^{n}\right) \rightarrow L^{n p /(n-2|s| p)}\left(\mathbb{R}^{n}\right), \quad 1<p<\frac{n}{2|s|} .
$$

$$
\left\|(-\Delta)^{s} u\right\|_{H^{r-2 s}}=\left\|\mathscr{F}^{-1}\left\{m(\xi)\langle\xi\rangle^{r} \hat{u}(\xi)\right\}\right\|_{L^{2}},
$$

where $m(\xi)=\langle\xi\rangle^{-2 s}|\xi|^{2 s}$ is bounded and hence a Fourier multiplier on $L^{2}$, showing that $\left\|(-\Delta)^{s} u\right\|_{H^{r-2 s}} \leq$ $C\|u\|_{H^{r}}$. The second statement is the Hardy-Littlewood-Sobolev inequality [Hörmander 1983, Theorem 4.5.3].

Remark 2.2. If $s \geq 0$, the fractional Laplacian also extends as a bounded map

$$
\begin{aligned}
& (-\Delta)^{s}: W^{r, p}\left(\mathbb{R}^{n}\right) \rightarrow W^{r-2 s, p}\left(\mathbb{R}^{n}\right), \\
& (-\Delta)^{s}: C_{*}^{r}\left(\mathbb{R}^{n}\right) \rightarrow C_{*}^{r-2 s}\left(\mathbb{R}^{n}\right)
\end{aligned}
$$

whenever $r \in \mathbb{R}$ and $1<p<\infty$, where $W^{r, p}$ are the usual $L^{p}$ Sobolev (Bessel potential) spaces and $C_{*}^{r}$ are the Zygmund spaces; see [Taylor 1996]. An even larger domain for $(-\Delta)^{s}$ is obtained as in [Silvestre 2007] by considering the test function space

$$
\mathscr{S}_{s}=\left\{u \in C^{\infty}\left(\mathbb{R}^{n}\right):\langle\cdot\rangle^{n+2 s} \partial^{\alpha} u \in L^{\infty}\left(\mathbb{R}^{n}\right) \text { for any multi-index } \alpha\right\},
$$

equipped with the topology induced by the seminorms $\left\|\langle\cdot\rangle^{n+2 s} \partial^{\alpha} u\right\|_{L^{\infty}}$. Then $(-\Delta)^{s}$ is continuous from $\mathscr{S}$ to $\mathscr{S}_{s}$ and extends to the dual

$$
\mathscr{S}_{s}^{\prime}=\left\{u \in \mathscr{S}^{\prime}\left(\mathbb{R}^{n}\right): u=\sum_{|\alpha| \leq m} \partial^{\alpha} u_{\alpha} \text { for some } m \geq 0 \text { and } u_{\alpha} \in\langle\cdot\rangle^{n+2 a} L^{\infty}\left(\mathbb{R}^{n}\right)\right\} .
$$

However, in this article it suffices to work with the spaces $H^{s}\left(\mathbb{R}^{n}\right)$. 
2C. Dirichlet problem. Next we restrict our attention to nonlocal operators

$$
(-\Delta)^{s}, \quad 0<s<1,
$$

and consider the solvability of the Dirichlet problem

$$
\begin{array}{rlrl}
\left((-\Delta)^{s}+q\right) u & =F & & \text { in } \Omega, \\
u=f & & \text { in } \Omega_{e},
\end{array}
$$

where, for a bounded open set $\Omega \subset \mathbb{R}^{n}$, we denote the exterior domain by $\Omega_{e}=\mathbb{R}^{n} \backslash \bar{\Omega}$. Here $F$ may be a function in $\Omega$, or more generally an element of $\left(\widetilde{H}^{s}(\Omega)\right)^{*}$. We also denote the restriction to $\Omega$ by

$$
r_{\Omega} u=\left.u\right|_{\Omega},
$$

and if $U \subset \mathbb{R}^{n}$ is open and $u, v \in L^{2}(U)$ we write

$$
(u, v)_{U}=\int_{U} u v d x .
$$

Lemma 2.3. Let $\Omega \subset \mathbb{R}^{n}$ be a bounded open set, let $0<s<1$, and let $q \in L^{\infty}(\Omega)$. Let $B_{q}$ be the bilinear form defined for $v, w \in H^{s}\left(\mathbb{R}^{n}\right)$ by

$$
B_{q}(v, w)=\left((-\Delta)^{s / 2} v,(-\Delta)^{s / 2} w\right)_{\mathbb{R}^{n}}+\left(q r_{\Omega} v, r_{\Omega} w\right)_{\Omega} .
$$

(a) There is a countable set $\Sigma=\left\{\lambda_{j}\right\}_{j=1}^{\infty} \subset \mathbb{R}, \lambda_{1} \leq \lambda_{2} \leq \cdots \rightarrow \infty$, with the following property: if $\lambda \in \mathbb{R} \backslash \Sigma$, then for any $F \in\left(\widetilde{H}^{s}(\Omega)\right)^{*}$ and $f \in H^{s}\left(\mathbb{R}^{n}\right)$ there is a unique $u \in H^{s}\left(\mathbb{R}^{n}\right)$ satisfying

$$
B_{q}(u, w)-\lambda(u, w)_{\mathbb{R}^{n}}=F(w) \quad \text { for } w \in \widetilde{H}^{s}(\Omega), \quad u-f \in \widetilde{H}^{s}(\Omega) .
$$

One has the norm estimate

$$
\|u\|_{H^{s}\left(\mathbb{R}^{n}\right)} \leq C\left(\|F\|_{\left(\widetilde{H}^{s}(\Omega)\right)^{*}}+\|f\|_{H^{s}\left(\mathbb{R}^{n}\right)}\right),
$$

with $C$ independent of $F$ and $f$.

(b) The function $u$ in (a) is also the unique $u \in H^{s}\left(\mathbb{R}^{n}\right)$ satisfying

$$
r_{\Omega}\left((-\Delta)^{s}+q-\lambda\right) u=F \quad \text { in the sense of distributions in } \Omega
$$

and $u-f \in \widetilde{H}^{s}(\Omega)$.

(c) One has $0 \notin \Sigma$ if (1-1) holds. If $q \geq 0$, then one has $\Sigma \subset(0, \infty)$ and (1-1) always holds.

Proof. The proof is standard, but we give the details for completeness.

(a) If $u=f+v$, it is enough to find $v \in \widetilde{H}^{s}(\Omega)$ solving the equivalent problem

$$
B_{q}(v, w)-\lambda(v, w)_{\mathbb{R}^{n}}=\widetilde{F}(w), \quad w \in \widetilde{H}^{s}(\Omega),
$$

for a suitable $\widetilde{F} \in\left(\widetilde{H}^{s}(\Omega)\right)^{*}$. Consider the bilinear form $B_{q}(v, w)$ for $v, w \in \widetilde{H}^{s}(\Omega)$. If $\mu>\left\|q_{-}\right\|_{L^{\infty}(\Omega)}$, where $q_{-}(x)=-\min \{0, q(x)\}$, then, for $v \in \widetilde{H}^{s}(\Omega)$,

$$
B_{q}(v, v)+\mu(v, v)_{\mathbb{R}^{n}} \geq\left\|(-\Delta)^{s / 2} v\right\|_{L^{2}}^{2}+\left(\mu-\left\|q_{-}\right\|_{L^{\infty}(\Omega)}\right)\|v\|_{L^{2}}^{2} \geq c\|v\|_{H^{s}}^{2} .
$$


By the Riesz representation theorem, there is a unique $v=G_{\mu} \widetilde{F}$ in $\widetilde{H}^{s}(\Omega)$ satisfying $B_{q}(v, w)+$ $\mu(v, w)_{\mathbb{R}^{n}}=\widetilde{F}(w)$ for $w \in \widetilde{H}^{s}(\Omega)$. Now

$$
B_{q}(v, \cdot)-\lambda(v, \cdot)=\widetilde{F}(\cdot) \text { on } \widetilde{H}^{s}(\Omega) \quad \Longleftrightarrow \quad v=G_{\mu}[(\mu+\lambda) v+\widetilde{F}] .
$$

The operator $G_{\mu}$ is bounded $\left(\widetilde{H}^{s}(\Omega)\right)^{*} \rightarrow \widetilde{H}^{s}(\Omega)$, and by compact Sobolev embedding it gives rise to a compact, self-adjoint, positive definite operator $L^{2}(\Omega) \rightarrow L^{2}(\Omega)$. The spectral theorem for compact self-adjoint operators proves (a); in particular the eigenvalues of $G_{\mu}$ are $\left\{1 /\left(\lambda_{j}+\mu\right)\right\}_{j=1}^{\infty}$, and $\Sigma \subset$ $\left[-\left\|q_{-}\right\|_{L^{\infty}}, \infty\right)$. In fact $\Sigma \subset\left(-\left\|q_{-}\right\|_{L^{\infty}}, \infty\right)$, since otherwise there would be a nontrivial function $u \in \widetilde{H}^{s}(\Omega)$ with $B_{q}(u, u)+\left\|q_{-}\right\|_{L^{\infty}}(u, u)_{\mathbb{R}^{n}}=0$, showing that $(-\Delta)^{s / 2} u=0$ and thus $u \equiv 0$, which is a contradiction.

(b) If $u$ is as in (a), then clearly $u$ satisfies

$$
B_{q}(u, v)-\lambda(u, v)_{\mathbb{R}^{n}}=F(v) \quad \text { for } v \in C_{c}^{\infty}(\Omega), \quad u-f \in \widetilde{H}^{s}(\Omega),
$$

which is equivalent to the condition in (b). Conversely, if $u$ satisfies (2-1) for $v \in C_{c}^{\infty}(\Omega)$, then (2-1) holds for $v \in \widetilde{H}^{s}(\Omega)$ by density, and thus $u$ is the unique solution in (a).

(c) Note that (1-1) states that any solution in $H_{\bar{\Omega}}^{s}$ is identically zero. This is stronger than stating that any solution in $\widetilde{H}^{s}(\Omega)$ is zero, which is equivalent to $0 \notin \Sigma$ by the Fredholm alternative. If $q \geq 0$, then it was proved in (a) that $\Sigma \subset(0, \infty)$ and thus (1-1) holds.

DN map. By analogy with the case $s=1$, we may define the DN map for the fractional Schrödinger equation via the bilinear form $B_{q}$ for the equation given in Lemma 2.3.

Lemma 2.4. Let $\Omega \subset \mathbb{R}^{n}$ be a bounded open set, let $0<s<1$, and let $q \in L^{\infty}(\Omega)$ satisfy (1-1). There is a bounded linear map

$$
\Lambda_{q}: X \rightarrow X^{*}
$$

where $X$ is the abstract trace space $X=H^{s}\left(\mathbb{R}^{n}\right) / \widetilde{H}^{s}(\Omega)$, defined by

$$
\left(\Lambda_{q}[f],[g]\right)=B_{q}\left(u_{f}, g\right), \quad f, g \in H^{s}\left(\mathbb{R}^{n}\right),
$$

where $u_{f} \in H^{s}\left(\mathbb{R}^{n}\right)$ solves $\left((-\Delta)^{s}+q\right) u=0$ in $\Omega$ with $u-f \in \widetilde{H}^{s}(\Omega)$. One has

$$
\left(\Lambda_{q}[f],[g]\right)=\left([f], \Lambda_{q}[g]\right), \quad f, g \in H^{s}\left(\mathbb{R}^{n}\right) .
$$

Proof. Let $f, g \in H^{s}\left(\mathbb{R}^{n}\right)$. Since $B_{q}\left(u_{f+\varphi}, g+\psi\right)=B_{q}\left(u_{f}, g\right)$ for $\varphi, \psi$ in $\widetilde{H}^{s}(\Omega)$, the expression $\left(\Lambda_{q}[f],[g]\right)=B_{q}\left(u_{f}, g\right)$ is well-defined and

$$
\begin{aligned}
\left|\left(\Lambda_{q}[f],[g]\right)\right| & \leq\left\|(-\Delta)^{s / 2} u_{f}\right\|_{L^{2}}\left\|(-\Delta)^{s / 2} g\right\|_{L^{2}}+\|q\|_{L^{\infty}}\left\|u_{f}\right\|_{L^{2}}\|g\|_{L^{2}} \\
& \leq C\left\|u_{f}\right\|_{H^{s}}\|g\|_{H^{s}} \leq C\|f\|_{H^{s}}\|g\|_{H^{s}} .
\end{aligned}
$$

Thus $\left|\left(\Lambda_{q}[f],[g]\right)\right| \leq C\|[f]\|_{X}\|[g]\|_{X}$, so $\Lambda_{q}$ is well-defined and bounded, and self-adjointness follows by taking $g=u_{g}$. 
If $\Omega$ has Lipschitz boundary, then $X=H^{s}\left(\Omega_{e}\right)$ and $X^{*}=H_{\bar{\Omega}_{e}}^{-s}$ with natural identifications, but functions in $H_{\bar{\Omega}_{e}}^{-s}$ are only uniquely determined by their restrictions to $\Omega_{e}$ if $s<\frac{1}{2}$. Thus, for Lipschitz domains, one should think of the DN map as an operator

$$
\Lambda_{q}: H^{s}\left(\Omega_{e}\right) \rightarrow H_{\bar{\Omega}_{e}}^{-s}\left(\mathbb{R}^{n}\right) .
$$

The integral identity that allows us to solve the inverse problem is a direct consequence of Lemma 2.4. For simplicity, we will write $f$ instead of $[f]$ for elements of $X$.

Lemma 2.5. Let $\Omega \subset \mathbb{R}^{n}$ be a bounded open set, let $0<s<1$, and let $q_{1}, q_{2} \in L^{\infty}(\Omega)$ satisfy (1-1). For any $f_{1}, f_{2} \in X$ one has

$$
\left(\left(\Lambda_{q_{1}}-\Lambda_{q_{2}}\right) f_{1}, f_{2}\right)=\left(\left(q_{1}-q_{2}\right) r_{\Omega} u_{1}, r_{\Omega} u_{2}\right)_{\Omega},
$$

where $u_{j} \in H^{s}\left(\mathbb{R}^{n}\right)$ solves $\left((-\Delta)^{s}+q_{j}\right) u_{j}=0$ in $\Omega$ with $\left.u_{j}\right|_{\Omega_{e}}=f_{j}$.

Proof. One has

$$
\begin{aligned}
\left(\left(\Lambda_{q_{1}}-\Lambda_{q_{2}}\right) f_{1}, f_{2}\right) & =\left(\Lambda_{q_{1}} f_{1}, f_{2}\right)-\left(f_{1}, \Lambda_{q_{2}} f_{2}\right)=B_{q_{1}}\left(u_{1}, u_{2}\right)-B_{q_{2}}\left(u_{1}, u_{2}\right) \\
& =\left(\left(q_{1}-q_{2}\right) r_{\Omega} u_{1}, r_{\Omega} u_{2}\right)_{\Omega} .
\end{aligned}
$$

\section{Uniqueness properties}

We prove the uniqueness result for the fractional Laplacian, Theorem 1.2, which is an easy consequence of the Carleman estimates in [Rüland 2015] and the Caffarelli-Silvestre extension [2007].

Proof of Theorem 1.2. Assume first that $u$ is a continuous bounded function in $\mathbb{R}^{n}$. Write $\mathbb{R}_{+}^{n+1}=\{(x, y)$ : $\left.x \in \mathbb{R}^{n}, y>0\right\}$, and denote by $w$ the extension of $u$ to $\mathbb{R}_{+}^{n+1}$ defined by

$$
w(x, y)=\left(P_{y} * u\right)(x), \quad P_{y}(x)=c_{n, s} \frac{y^{2 s}}{\left(|x|^{2}+y^{2}\right)^{(n+2 s) / 2}} .
$$

By [Cabré and Sire 2014, Remark 3.8], $w$ is the unique continuous bounded solution in $\overline{\mathbb{R}}^{n+1}$ of the Dirichlet problem

$$
\operatorname{div}\left(y^{1-2 s} \nabla w\right)=0 \quad \text { in } \mathbb{R}^{n+1},\left.\quad w\right|_{y=0}=u .
$$

If we additionally assume that $u \in H^{s}\left(\mathbb{R}^{n}\right)$, then by [Cabré and Sire 2014, Section 3] the solution $w$ satisfies $\int_{\mathbb{R}_{+}^{n+1}} y^{1-2 s}|\nabla w|^{2} d x d y<\infty$, and one has

$$
(-\Delta)^{s} u=-d_{s} \lim _{y \rightarrow 0^{+}} y^{1-2 s} \partial_{y} w(\cdot, y),
$$

where the limit exists in $H^{-s}\left(\mathbb{R}^{n}\right)$. See [Cabré and Sire 2014] for the precise values of the constants $c_{n, s}$ and $d_{s}$.

Assume now that $u$ is a continuous bounded function in $\mathbb{R}^{n}$ with $u \in H^{s}\left(\mathbb{R}^{n}\right)$, and $\left.u\right|_{W}=\left.(-\Delta)^{s} u\right|_{W}=0$, where $W$ is a ball in $\mathbb{R}^{n}$. Denote by $B$ the ball in $\mathbb{R}^{n+1}$ with $B \cap\{y=0\}=W$, and define $B^{+}=\{(x, y) \in B$ : $y>0\}$. Since $\left.u\right|_{W}=\left.(-\Delta)^{s} u\right|_{W}=0, w$ satisfies

$$
\operatorname{div}\left(y^{1-2 s} \nabla w\right)=0 \quad \text { in } B^{+},\left.\quad w\right|_{B \cap\{y=0\}}=\left.\lim _{y \rightarrow 0^{+}} y^{1-2 s} \partial_{y} w\right|_{B \cap\{y=0\}}=0 .
$$


The function $w$ thus satisfies the conditions in [Rüland 2015, Proposition 2.2], and one obtains that $\left.w\right|_{B^{+}} \equiv 0$. But $w$ is real-analytic in $\mathbb{R}_{+}^{n+1}$ as the solution of an elliptic equation with real-analytic coefficients; see [Hörmander 1983, Theorem 8.6.1]. Hence $w \equiv 0$ in $\mathbb{R}^{n+1}$, which implies that $u \equiv 0$.

Finally, let $u \in H^{-r}\left(\mathbb{R}^{n}\right)$ for some $r>0$, and $\left.u\right|_{W}=\left.(-\Delta)^{s} u\right|_{W}=0$ for some ball $W \subset \mathbb{R}^{n}$. Consider the smooth approximations

$$
u_{\varepsilon}=u * \varepsilon^{-n} \varphi(\cdot / \varepsilon),
$$

where $\varphi \in C_{c}^{\infty}\left(\mathbb{R}^{n}\right)$ satisfies $\int \varphi d x=1, \varphi \geq 0$, and $\varphi=0$ for $|x| \geq 1$. There exist $\varepsilon_{0}>0$ and a smaller ball $W^{\prime} \subset W$ such that $\left.u_{\varepsilon}\right|_{W^{\prime}}=0$ and also $\left.(-\Delta)^{s} u_{\varepsilon}\right|_{W^{\prime}}=\left.\left((-\Delta)^{s} u\right) * \varepsilon^{-n} \varphi(\cdot / \varepsilon)\right|_{W^{\prime}}=0$ whenever $\varepsilon<\varepsilon_{0}$. Now each $u_{\varepsilon}$ is in $H^{\alpha}\left(\mathbb{R}^{n}\right)$ for any $\alpha \in \mathbb{R}$, since $\hat{u}_{\varepsilon}(\xi)=m(\xi) \hat{u}(\xi)$, where $m(\xi)=\hat{\varphi}(\varepsilon \xi)$ is a Schwartz function and $\langle\xi\rangle^{-r} \hat{u}(\xi)$ is in $L^{2}$. By Sobolev embedding, each $u_{\varepsilon}$ is also continuous and bounded in $\mathbb{R}^{n}$. The argument above implies that $u_{\varepsilon} \equiv 0$ whenever $\varepsilon<\varepsilon_{0}$, showing that $u=\lim _{\varepsilon \rightarrow 0} u_{\varepsilon}=0$.

Remark 3.1. We note that for $s=\frac{1}{2}$ the above argument simplifies: the function $w$ in the proof is just the harmonic extension of $u$ to $\mathbb{R}^{n+1}$, and it satisfies $\left.w\right|_{W \times\{y=0\}}=\left.\partial_{y} w\right|_{W \times\{y=0\}}=0$. The odd extension $\tilde{w}$ of $w$ to $W \times \mathbb{R}$ is smooth, satisfies $\Delta_{x, y} \tilde{w}=0$, and $\left.\tilde{w}\right|_{W \times\{y=0\}}=\left.\partial_{y} \tilde{w}\right|_{W \times\{y=0\}}=0$. Using the equation one observes that $\tilde{w}$ vanishes to infinite order on $W \times\{y=0\}$; thus by analyticity $\tilde{w} \equiv 0$ and $u \equiv 0$.

Remark 3.2. For comparison, we recall the original argument in [Riesz 1938, Chapitre III.11] for proving a result like Theorem 1.2. There are two steps: first one uses the Kelvin transform to reduce to the case where $u$ and $(-\Delta)^{s} u$ vanish outside some ball, and then one computes derivatives of $u$ and lets $x \rightarrow \infty$ to show that all moments of $(-\Delta)^{s} u$ must vanish. See [Isakov 1990, Lemma 3.5.4] for another proof of the second step.

Let $u$ be in the Sobolev space $W^{-r, q}\left(\mathbb{R}^{n}\right)$ for some $r \in \mathbb{R}$, where $q=2 n /(n+2 s)$. By approximation, translation and scaling, we may assume that $u \in W^{t, q}\left(\mathbb{R}^{n}\right)$ for any $t>0$ and $\left.u\right|_{B}=\left.(-\Delta)^{s} u\right|_{B}=0$, where $B$ is the unit ball. Write $f=(-\Delta)^{s} u$, so $f, u \in L^{q} \cap L^{\infty}$ and $u=I_{2 s} f$. Define

$$
v=R_{2 s} u, \quad g=R_{-2 s} f,
$$

where $R_{\alpha} f(x)=|x|^{\alpha-n} f(K(x))$ and $K(x)=x /|x|^{2}$ is the Kelvin transform. Since det $D K(x)=-|x|^{-2 n}$ and $|K(x)-K(y)|=|x-y| /(|x||y|)$, one computes $\left\|R_{-2 s} f\right\|_{L^{q}}=\|f\|_{L^{q}}$ and $R_{2 s} I_{2 s} f=I_{2 s} R_{-2 s} f$. Then $g \in L^{q}$, both $v=I_{2 s} g$ and $g$ vanish outside $B$, and

$$
v(x)=c_{n, s} \int_{B}|x-y|^{2 s-n} g(y) d y=0, \quad|x|>1 .
$$

In particular, letting $x \rightarrow \infty$, one gets $\int_{B} g(y) d y=0$. Applying powers of the Laplacian to $v(x)$ we get

$$
\int_{B}|x-y|^{2 s-n-2 k} g(y) d y=0, \quad k \geq 0,|x|>1 .
$$

Computing $\partial_{x_{j}} v(x)$ and letting $x \rightarrow \infty$ gives $\int_{B} y_{j} g(y) d y=0$. Repeating this for higher-order derivatives implies that $\int_{B} y^{\alpha} g(y) d y=0$ for any multi-index $\alpha$; hence $g \equiv 0$. This finally gives $f \equiv 0$ and $u \equiv 0$.

The above argument seems to require that $f \in L^{q}$ for $q$ close to 1 in order for $R_{-2 s} f$ to be an $L^{p}$ function for some $p$. If one starts with a solution $u \in H^{-r}$ for some $r$ (as stated in Theorem 1.2), after 
approximation one gets $f \in L^{2} \cap L^{\infty}$ and then there is an issue since $R_{-2 s} f$ might have a nonintegrable singularity at 0 . Thus it seems that this method is not sufficient for proving Theorem 1.2 in full generality.

\section{Approximation in $L^{2}(\Omega)$}

We will use the following Runge approximation property for solutions of the fractional Schrödinger equation. If $q \in L^{\infty}(\Omega)$ satisfies (1-1), we denote by $P_{q}$ the Poisson operator

$$
P_{q}: X \rightarrow H^{s}\left(\mathbb{R}^{n}\right), \quad f \mapsto u,
$$

where $X=H^{s}\left(\mathbb{R}^{n}\right) / \widetilde{H}^{s}(\Omega)$ is the abstract space of exterior values, and $u \in H^{s}\left(\mathbb{R}^{n}\right)$ is the unique solution of $\left((-\Delta)^{s}+q\right) u=0$ in $\Omega$ with $u-f \in \widetilde{H}^{s}(\Omega)$ given in Lemma 2.3.

Lemma 4.1. Let $\Omega \subset \mathbb{R}^{n}$ be bounded open set, let $0<s<1$, and let $q \in L^{\infty}(\Omega)$ satisfy (1-1). Let also $W$ be any open subset of $\Omega_{e}$. Consider the set

Then $\mathcal{R}$ is dense in $L^{2}(\Omega)$.

$$
\mathcal{R}=\left\{\left.u\right|_{\Omega}: u=P_{q} f, f \in C_{c}^{\infty}(W)\right\} .
$$

Proof. By the Hahn-Banach theorem, it is enough to show that any $v \in L^{2}(\Omega)$ with $(v, w)_{\Omega}=0$ for all $w \in \mathcal{R}$ must satisfy $v \equiv 0$. If $v$ is such a function, then

$$
\left(v, r_{\Omega} P_{q} f\right)_{\Omega}=0, \quad f \in C_{c}^{\infty}(W) .
$$

We claim that the formal adjoint of $r_{\Omega} P_{q}$ is given by

$$
\left(v, r_{\Omega} P_{q} f\right)_{\Omega}=-B_{q}(\varphi, f), \quad f \in C_{c}^{\infty}(W),
$$

where $\varphi \in H^{s}\left(\mathbb{R}^{n}\right)$ is the solution given by Lemma 2.3 of

$$
\left((-\Delta)^{s}+q\right) \varphi=v \quad \text { in } \Omega, \quad \varphi \in \widetilde{H}^{s}(\Omega) .
$$

In other words, $B_{q}(\varphi, w)=\left(v, r_{\Omega} w\right)_{\Omega}$ for any $w \in \widetilde{H}^{s}(\Omega)$. To prove (4-3), let $f \in C_{c}^{\infty}(W)$, and let $u_{f}=P_{q} f \in H^{s}\left(\mathbb{R}^{n}\right)$ so $u_{f}-f \in \widetilde{H}^{s}(\Omega)$. Then

$$
\left(v, r_{\Omega} P_{q} f\right)_{\Omega}=\left(v, r_{\Omega}\left(u_{f}-f\right)\right)_{\Omega}=B_{q}\left(\varphi, u_{f}-f\right)=-B_{q}(\varphi, f) .
$$

In the last line, we used that $u_{f}$ is a solution and $\varphi \in \widetilde{H}^{s}(\Omega)$.

Combining (4-2) and (4-3), we have

$$
B_{q}(\varphi, f)=0, \quad f \in C_{c}^{\infty}(W) .
$$

Since $r_{\Omega} f=0$, this implies

$$
0=\left((-\Delta)^{s / 2} \varphi,(-\Delta)^{s / 2} f\right)_{\mathbb{R}^{n}}=\left((-\Delta)^{s} \varphi, f\right)_{\mathbb{R}^{n}}, \quad f \in C_{c}^{\infty}(W) .
$$

In particular, $\varphi \in H^{s}\left(\mathbb{R}^{n}\right)$ satisfies

$$
\left.\varphi\right|_{W}=\left.(-\Delta)^{s} \varphi\right|_{W}=0 .
$$

Theorem 1.2 implies that $\varphi \equiv 0$, and thus also $v \equiv 0$. 


\section{Inverse problem}

It is now easy to prove the uniqueness result for the inverse problem.

Proof of Theorem 1.1. Note that if $F \in X^{*}$, then $\left.F\right|_{W_{2}}$ is a distribution in $W_{2}$ with $\left.F\right|_{W_{2}}(\varphi)=F([\varphi])$, $\varphi \in C_{c}^{\infty}\left(W_{2}\right)$. Now if $\left.\Lambda_{q_{1}} f\right|_{W_{2}}=\left.\Lambda_{q_{2}} f\right|_{W_{2}}$ for any $f \in C_{c}^{\infty}\left(W_{1}\right)$, the integral identity in Lemma 2.5 yields that

$$
\int_{\Omega}\left(q_{1}-q_{2}\right) u_{1} u_{2} d x=0
$$

whenever $u_{j} \in H^{s}\left(\mathbb{R}^{n}\right)$ solve $\left((-\Delta)^{s}+q_{j}\right) u_{j}=0$ in $\Omega$ with exterior values in $C_{c}^{\infty}\left(W_{j}\right)$. Let $h \in L^{2}(\Omega)$, and use the approximation result, Lemma 4.1, to find sequences $\left(u_{j}^{(k)}\right)$ of functions in $H^{s}\left(\mathbb{R}^{n}\right)$ that satisfy

$$
\begin{gathered}
\left((-\Delta)^{s}+q_{1}\right) u_{1}^{(k)}=\left((-\Delta)^{s}+q_{2}\right) u_{2}^{(k)}=0 \quad \text { in } \Omega, \\
u_{j}^{(k)} \text { have exterior values in } C_{c}^{\infty}\left(W_{j}\right), \\
r_{\Omega} u_{1}^{(k)}=h+r_{1}^{(k)}, \quad r_{\Omega} u_{2}^{(k)}=1+r_{2}^{(k)},
\end{gathered}
$$

where $r_{1}^{(k)}, r_{2}^{(k)} \rightarrow 0$ in $L^{2}(\Omega)$ as $k \rightarrow \infty$. Inserting these solutions in the integral identity and taking the limit as $k \rightarrow \infty$ implies

$$
\int_{\Omega}\left(q_{1}-q_{2}\right) h d x=0
$$

Since $h \in L^{2}(\Omega)$ was arbitrary, we conclude that $q_{1}=q_{2}$.

\section{Higher-order approximation}

We proceed to prove Theorem 1.3(b). The argument is similar to that in Section 4, but since the approximation is in high regularity spaces, by duality we will need to solve Dirichlet problems with data in negative-order Sobolev spaces. This follows again by duality from regularity results for the Dirichlet problem proved in [Hörmander 1965; Grubb 2015].

We will next introduce function spaces from [Grubb 2015]. Note that the smoothness indices $s$ and $s(r)$ in this article correspond to $a$ and $a(s)$ in [Grubb 2015]. Assume that $\Omega \subset \mathbb{R}^{n}$ is a bounded domain with $C^{\infty}$ boundary, and let $q \in C_{c}^{\infty}(\Omega)$ satisfy the analogue of (1-1),

$$
\text { if } u \in H^{s}\left(\mathbb{R}^{n}\right) \text { solves }\left((-\Delta)^{s}+q\right) u=0 \text { in } \Omega \text { and }\left.u\right|_{\Omega_{e}}=0 \text {, then } u \equiv 0 \text {. }
$$

We assume $q$ compactly supported to fit the operator theory in [Grubb 2015]. Define

$$
\mathcal{E}_{s}(\bar{\Omega})=e^{+} d(x)^{s} C^{\infty}(\bar{\Omega}),
$$

where $e^{+}$denotes extension by zero from $\Omega$ to $\mathbb{R}^{n}$, and $d$ is a $C^{\infty}$ function in $\bar{\Omega}$, positive in $\Omega$, and satisfying $d(x)=\operatorname{dist}(x, \partial \Omega)$ near $\partial \Omega$. If $r>s-\frac{1}{2}$ we will also consider the Banach space $H^{s(r)}(\bar{\Omega})$ which arises as the exact solution space of functions $u$ satisfying

$$
r_{\Omega}\left((-\Delta)^{s}+q\right) u \in H^{r-2 s}(\Omega),\left.\quad u\right|_{\Omega_{e}}=0 .
$$


We will not give the actual definition, but instead we will use the following properties from [Grubb 2015].

Lemma 6.1. For any $r>s-\frac{1}{2}$, there is a Banach space $H^{s(r)}(\bar{\Omega})$ with the following properties:

(a) $H^{s(r)}(\bar{\Omega}) \subset H_{\bar{\Omega}}^{s-1 / 2}$ with continuous inclusion.

(b) $H^{s(r)}(\bar{\Omega})=H_{\bar{\Omega}}^{r}$ if $r \in\left(s-\frac{1}{2}, s+\frac{1}{2}\right)$.

(c) The operator $r_{\Omega}\left((-\Delta)^{s}+q\right)$ is a homeomorphism from $H^{s(r)}(\bar{\Omega})$ onto $H^{r-2 s}(\Omega)$.

(d) $H_{\bar{\Omega}}^{r} \subset H^{s(r)}(\bar{\Omega}) \subset H_{\mathrm{loc}}^{r}(\Omega)$ with continuous inclusions; i.e., multiplication by any $\chi \in C_{c}^{\infty}(\Omega)$ is bounded $H^{s(r)}(\bar{\Omega}) \rightarrow H^{r}(\Omega)$.

(e) $\mathcal{E}_{s}(\bar{\Omega})=\bigcap_{r>s-1 / 2} H^{s(r)}(\bar{\Omega})$, and $\mathcal{E}_{s}(\bar{\Omega})$ is dense in $H^{s(r)}(\bar{\Omega})$.

Proof. Parts (a) and (b) follow from [Grubb 2015, Section 1]. Part (c) follows since $r_{\Omega}\left((-\Delta)^{s}+q\right)$ : $H^{s(r)}(\bar{\Omega}) \rightarrow H^{r-2 s}(\Omega)$ is a Fredholm operator [Grubb 2015, Theorem 2], it has a finite-dimensional kernel and range complement independent of $r$ [Grubb 2014, Theorem 3.5], and for $r=s$ the kernel and range complement are trivial using (6-1) and Lemma 2.3. Part (d) follows from (c) and (a), or alternatively from the definitions in [Grubb 2015, Section 1]. Part (e) is in [Grubb 2015, Proposition 4.1].

We next prove an approximation result in the space $\mathcal{E}_{s}(\bar{\Omega})$, equipped with the topology induced by the norms $\left\{\|\cdot\|_{H^{s(m)}(\bar{\Omega})}\right\}_{m=1}^{\infty}$. Then $\mathcal{E}_{s}(\bar{\Omega})$ is a Fréchet space.

Lemma 6.2. Let $\Omega \subset \mathbb{R}^{n}$ be a bounded domain with $C^{\infty}$ boundary, let $0<s<1$, let $W$ be an open subset of $\Omega_{e}$, and let $q \in C_{c}^{\infty}(\Omega)$ satisfy (6-1). If $P_{q}$ is the Poisson operator in (4-1), define

$$
\mathcal{R}=\left\{e^{+} r_{\Omega} P_{q} f: f \in C_{c}^{\infty}(W)\right\} .
$$

Then $\mathcal{R}$ is a dense subset of $\mathcal{E}_{S}(\bar{\Omega})$.

Proof. Note that $\mathcal{R} \subset \mathcal{E}_{s}(\bar{\Omega})$, since for $f \in C_{c}^{\infty}(W)$ one has $P_{q} f=f+v$, where $r_{\Omega}\left((-\Delta)^{s}+q\right) v \in C^{\infty}(\bar{\Omega})$ and $\left.v\right|_{\Omega_{e}}=0$; hence $v \in \mathcal{E}_{s}(\bar{\Omega})$ by Lemma 6.1 .

Let $L$ be a continuous linear functional on $\mathcal{E}_{s}(\bar{\Omega})$ that satisfies

$$
L\left(e^{+} r_{\Omega} P_{q} f\right)=0, \quad f \in C_{c}^{\infty}(W) .
$$

It is enough to show that $L \equiv 0$, since then $\mathcal{R}$ will be dense by the Hahn-Banach theorem.

By the properties of Fréchet spaces, there exists an integer $r$ so that

$$
|L(u)| \leq C \sum_{m=1}^{r}\|u\|_{H^{s(m)}(\bar{\Omega})} \leq C^{\prime}\|u\|_{H^{s(r)}(\bar{\Omega})}, \quad u \in \mathcal{E}_{s}(\bar{\Omega}) .
$$

Since $\mathcal{E}_{s}(\bar{\Omega})$ is dense in $H^{s(r)}(\bar{\Omega})$, we know $L$ has a unique bounded extension $\bar{L} \in\left(H^{s(r)}(\bar{\Omega})\right)^{*}$. Consider next the homeomorphism in Lemma 6.1,

$$
T=r_{\Omega}\left((-\Delta)^{s}+q\right): H^{s(r)}(\bar{\Omega}) \rightarrow H^{r-2 s}(\Omega) .
$$

Its adjoint is a bounded map between the dual Banach spaces,

$$
T^{*}:\left(H^{r-2 s}(\Omega)\right)^{*} \rightarrow\left(H^{s(r)}(\bar{\Omega})\right)^{*} .
$$


The map $T^{*}$ is also a homeomorphism, with inverse given by $\left(T^{-1}\right)^{*}$. Using the identification

$$
\left(H^{r-2 s}(\Omega)\right)^{*}=H_{\bar{\Omega}}^{-r+2 s}
$$

one has

$$
T^{*} v(w)=(v, T w), \quad w \in H^{s(r)}(\bar{\Omega}) .
$$

Now let $v \in H_{\bar{\Omega}}^{-r+2 s}$ be the unique function satisfying $T^{*} v=\bar{L}$, and choose a sequence $\left(v_{j}\right)_{j=1}^{\infty} \subset C_{c}^{\infty}(\Omega)$ with $v_{j} \rightarrow v$ in $H^{-r+2 s}$. If $f \in C_{c}^{\infty}(W)$, recall that $e^{+} r_{\Omega} P_{q} f=P_{q} f-f$, and observe that

$$
\begin{aligned}
0 & =L\left(e^{+} r_{\Omega} P_{q} f\right)=\bar{L}\left(P_{q} f-f\right)=T^{*} v\left(P_{q} f-f\right)=\left(v, T\left(P_{q} f-f\right)\right) \\
& =-(v, T f)=-\lim \left(v_{j},\left((-\Delta)^{s}+q\right) f\right)=-\lim \left(\left((-\Delta)^{s}+q\right) v_{j}, f\right) .
\end{aligned}
$$

Here we used that $T P_{q} f=0$ and $v_{j} \in C_{c}^{\infty}(\Omega)$. Since $f \in C_{c}^{\infty}(W)$, we may take the limit as $j \rightarrow \infty$ and obtain that

$$
\left((-\Delta)^{s} v, f\right)=0, \quad f \in C_{c}^{\infty}(W) .
$$

Thus $v \in H^{-r+2 s}\left(\mathbb{R}^{n}\right)$ satisfies

$$
\left.v\right|_{W}=\left.(-\Delta)^{s} v\right|_{W}=0 .
$$

By Theorem 1.2 it follows that $v \equiv 0$. This implies that $\bar{L} \equiv 0$ and $L \equiv 0$ as required.

Proof of Theorem 1.3. Let $\Omega \subset \Omega_{1} \subset \mathbb{R}^{n}$ be open sets with $\Omega_{1} \backslash \bar{\Omega} \neq \varnothing$ and $\Omega$ bounded. Since $\Omega_{1} \backslash \bar{\Omega} \neq \varnothing$, we may find a small ball $W$ with $\bar{W} \subset \Omega_{1} \backslash \bar{\Omega}$. Lemma 4.1 implies that any $f \in L^{2}(\Omega)$ can be approximated in $L^{2}(\Omega)$ by functions $\left.u\right|_{\Omega}$, where $u$ solves $\left((-\Delta)^{s}+q\right) u=0$ in $\Omega$ and $\operatorname{supp}(u) \subset \bar{\Omega} \cup \bar{W}$. Since $\bar{\Omega} \cup \bar{W} \subset \bar{\Omega}_{1}$, part (a) follows.

As for part (b), if $f \in C^{\infty}(\bar{\Omega})$ and if $g=e^{+} d(x)^{s} f \in \mathcal{E}_{s}(\bar{\Omega})$, Lemma 6.2 ensures that there is a sequence $\left(u_{j}\right)_{j=1}^{\infty} \subset H^{s}\left(\mathbb{R}^{n}\right)$ with

$$
\left((-\Delta)^{s}+q\right) u_{j}=0 \quad \text { in } \Omega, \quad \operatorname{supp}\left(u_{j}\right) \subset \bar{\Omega}_{1},
$$

so that $e^{+} r_{\Omega} u_{j} \in \mathcal{E}_{s}(\bar{\Omega})$ and

$$
e^{+} r_{\Omega} u_{j} \rightarrow g \text { in } \mathcal{E}_{s}(\bar{\Omega}) .
$$

The result will follow if we can show that

$$
M: C^{\infty}(\bar{\Omega}) \rightarrow \mathcal{E}_{s}(\bar{\Omega}), \quad M f=e^{+} d(x)^{s} f,
$$

is a homeomorphism, since then applying $M^{-1}=d(x)^{-s} r_{\Omega}$ gives

$$
d(x)^{-s} r_{\Omega} u_{j} \rightarrow f \quad \text { in } C^{\infty}(\bar{\Omega}) .
$$

But $M$ is a bijective linear map between Fréchet spaces and has closed graph: if $f_{j} \rightarrow f$ in $C^{\infty}$ and $M f_{j} \rightarrow h$ in $\mathcal{E}_{s}$, then also $M f_{j} \rightarrow M f$ in $L^{\infty}$ and one obtains $M f=h$ by uniqueness of distributional limits. Thus $M$ is a homeomorphism by the closed graph and open mapping theorems (in other words, there is a unique Fréchet space topology on $\mathcal{E}_{s}(\bar{\Omega})$ stronger than the Hausdorff topology inherited from $\mathcal{D}^{\prime}\left(\mathbb{R}^{n}\right)$ ). 
Remark 6.3. Let us note the following consequence of Theorem 1.3(b): if $k \geq 0$ and $R>1$ are fixed, then for any $g \in C^{k}\left(\bar{B}_{1}\right)$ and for any $\varepsilon>0$ there is a function $u \in H^{s}\left(\mathbb{R}^{n}\right)$ satisfying

$$
(-\Delta)^{s} u=0 \quad \text { in } B_{1}, \quad \operatorname{supp}(u) \subset \bar{B}_{R}, \quad\|u-g\|_{C^{k}\left(\bar{B}_{1}\right)}<\varepsilon .
$$

This can be seen by taking $\Omega=B_{r}$ and $\Omega_{1}=B_{R}$, where $1<r<R$, and by choosing $f \in C^{\infty}\left(\bar{B}_{r}\right)$ with $\left\|f-d(x)^{-s} g\right\|_{C^{k}\left(\bar{B}_{1}\right)}$ small enough.

\section{Appendix: The DN map}

The abstract definition of the DN map $\Lambda_{q}$ in Section 2 is sufficient for the formulation and solution of the inverse problem. However, in this appendix we will give more concrete descriptions of the DN map, valid under stronger regularity assumptions. For simplicity we assume that the boundary and the potential are $C^{\infty}$.

\section{DN map and $(-\Delta)^{s}$.}

Lemma A.1. Let $\Omega \subset \mathbb{R}^{n}$ be a bounded open set with $C^{\infty}$ boundary, let $0<s<1$, and let $q \in C_{c}^{\infty}(\Omega)$ satisfy (1-1). For any $\beta \geq 0$ satisfying $s-\frac{1}{2}<\beta<\frac{1}{2}$, the restriction of $\Lambda_{q}$ to $H^{s+\beta}\left(\Omega_{e}\right)$ is the map

$$
\Lambda_{q}: H^{s+\beta}\left(\Omega_{e}\right) \rightarrow H^{-s+\beta}\left(\Omega_{e}\right), \quad \Lambda_{q} f=\left.(-\Delta)^{s} u_{f}\right|_{\Omega_{e}}
$$

where $u_{f} \in H^{s+\beta}\left(\mathbb{R}^{n}\right)$ solves $\left((-\Delta)^{s}+q\right) u=0$ in $\Omega$ with $\left.u\right|_{\Omega_{e}}=f$.

Proof. First we use a result from [Vishik and Eskin 1965]; see also [Grubb 2015]: if $\beta \in\left[0, \frac{1}{2}\right)$, then for any $f \in H^{s+\beta}\left(\Omega_{e}\right)$ there is a unique $u=u_{f} \in H^{s+\beta}\left(\mathbb{R}^{n}\right)$ satisfying

$$
\left((-\Delta)^{s}+q\right) u=0 \quad \text { in } \Omega,\left.\quad u\right|_{\Omega_{e}}=f .
$$

In fact [Grubb 2015, Theorem 3.1] asserts Fredholm solvability for the inhomogeneous problem, but the result above can be reduced to this case by taking an $H^{s+\beta}$ extension of $f$ to $\mathbb{R}^{n}$, and Fredholm solvability implies unique solvability since the finite-dimensional kernel and range complement are independent of $\beta$ by [Grubb 2014, Theorem 3.5] and they are trivial when $\beta=0$ by Lemma 2.3.

Now for $f, g \in H^{s+\beta}\left(\Omega_{e}\right)$, with $\beta \in\left[0, \frac{1}{2}\right)$, let $u_{f} \in H^{s+\beta}\left(\mathbb{R}^{n}\right)$ be the solution obtained above and let $e_{g} \in H^{s+\beta}\left(\mathbb{R}^{n}\right)$ be some extension of $g$. Then, by definition,

$$
\begin{aligned}
\left(\Lambda_{q} f, g\right) & =\left((-\Delta)^{s / 2} u_{f},(-\Delta)^{s / 2} e_{g}\right)_{\mathbb{R}^{n}}+\left(q r_{\Omega} u_{f}, r_{\Omega} e_{g}\right)_{\Omega} \\
& =\left((-\Delta)^{s} u_{f}, e_{g}\right)_{\mathbb{R}^{n}}+\left(q r_{\Omega} u_{f}, r_{\Omega} e_{g}\right)_{\Omega}
\end{aligned}
$$

since $\left((-\Delta)^{s / 2} u,(-\Delta)^{s / 2} v\right)_{\mathbb{R}^{n}}=\left((-\Delta)^{s} u, v\right)_{\mathbb{R}^{n}}$ holds first for Schwartz functions by the Parseval identity, and then also for $u, v \in H^{s}\left(\mathbb{R}^{n}\right)$ by density.

It remains to show that whenever $\alpha \in\left(-\frac{1}{2}, \frac{1}{2}\right), u \in H^{-\alpha}\left(\mathbb{R}^{n}\right), v \in H^{\alpha}\left(\mathbb{R}^{n}\right)$, then

$$
(u, v)_{\mathbb{R}^{n}}=\left(r_{\Omega} u, r_{\Omega} v\right)_{\Omega}+\left(r_{\Omega_{e}} u, r_{\Omega_{e}} v\right)_{\Omega_{e}}
$$


in the sense of distributional pairings. If (A-1) is true, then the assumption $\beta \in\left(s-\frac{1}{2}, \frac{1}{2}\right)$ implies $(-\Delta)^{s} u_{f} \in H^{-s+\beta}\left(\mathbb{R}^{n}\right)$ with $-s+\beta \in\left(-\frac{1}{2}, \frac{1}{2}\right)$, and since $u_{f}$ is a solution in $\Omega$ one has

$$
\left(\Lambda_{q} f, g\right)=\left((-\Delta)^{s} u_{f}, e_{g}\right)_{\mathbb{B}^{n}}+\left(q r_{\Omega} u_{f}, r_{\Omega} e_{g}\right)_{\Omega}=\left(r_{\Omega_{e}}(-\Delta)^{s} u_{f}, g\right)_{\Omega_{e}}
$$

which concludes the proof.

To show (A-1), let $\chi_{\Omega}$ be the characteristic function of $\Omega$. This is a pointwise multiplier on $H^{\gamma}\left(\mathbb{R}^{n}\right)$ for $\gamma \in\left(-\frac{1}{2}, \frac{1}{2}\right)$ [Triebel 2002], and the same is true for $1-\chi_{\Omega}$. We may write $u=\chi_{\Omega} u+\left(1-\chi_{\Omega}\right) u$ and similarly for $v$, and then

$$
(u, v)_{\mathbb{R}^{n}}=\left(\chi_{\Omega} u, \chi_{\Omega} v\right)_{\mathbb{R}^{n}}+\left(\left(1-\chi_{\Omega}\right) u,\left(1-\chi_{\Omega}\right) v\right)_{\mathbb{R}^{n}},
$$

where the cross terms vanish first for Schwartz $u, v$ and then in general by density. Now $\chi_{\Omega} u$ is in $H_{\bar{\Omega}}^{-\alpha}$, and hence can be approximated by functions in $C_{c}^{\infty}(\Omega)$. Using similar approximations for the other functions and restricting to $\Omega$ and $\Omega_{e}$ implies (A-1).

DN map and $\mathcal{N}_{s}$. Several nonlocal Neumann boundary operators appear in the literature; see [Dipierro et al. 2017a; Grubb 2016]. We will relate $\Lambda_{q}$ to the nonlocal Neumann boundary operator $\mathcal{N}_{s}$ introduced in [Dipierro et al. 2017a], defined pointwise by

$$
\mathcal{N}_{s} u(x)=c_{n, s} \int_{\Omega} \frac{u(x)-u(y)}{|x-y|^{n+2 s}} d y, \quad x \in \Omega_{e} .
$$

The next lemma contains a definition that applies to Sobolev functions. The result states that knowing $\left.\Lambda_{q} f\right|_{W}$ for $f \in C_{c}^{\infty}(W)$ is equivalent to knowing $\left.\mathcal{N}_{s} u_{f}\right|_{W}$ for $f \in C_{c}^{\infty}(W)$, since $\left.\Lambda_{q} f\right|_{W}$ and $\left.\mathcal{N}_{s} u_{f}\right|_{W}$ only differ by quantities that do not depend on the unknown potential $q$.

Lemma A.2. Assume the conditions in Lemma A.1. One has

$$
\Lambda_{q} f=\mathcal{N}_{s} u_{f}-m f+\left.(-\Delta)^{s}\left(E_{0} f\right)\right|_{\Omega_{e}}, \quad f \in H^{s+\beta}\left(\Omega_{e}\right),
$$

where, for $\gamma>-\frac{1}{2}, \mathcal{N}_{s}$ is the map

$$
\mathcal{N}_{s}: H^{\gamma}\left(\mathbb{R}^{n}\right) \rightarrow H_{\mathrm{loc}}^{\gamma}\left(\Omega_{e}\right), \quad \mathcal{N}_{s} u=\left.m u\right|_{\Omega_{e}}+\left.(-\Delta)^{s}\left(\chi_{\Omega} u\right)\right|_{\Omega_{e}},
$$

where $m \in C^{\infty}\left(\Omega_{e}\right)$ is given by $m(x)=c_{n, s} \int_{\Omega} 1 /|x-y|^{n+2 s} d y$ and $\chi_{\Omega}$ is the characteristic function of $\Omega$. Also, $E_{0}$ is extension by zero. If $u \in L^{2}\left(\mathbb{R}^{n}\right)$, then $\mathcal{N}_{s} u \in L_{\text {loc }}^{2}\left(\Omega_{e}\right)$ is given a.e. by the formula (A-2).

Proof. If $u \in H^{\gamma}\left(\mathbb{R}^{n}\right)$ with $\gamma>-\frac{1}{2}$, then $\left.m u\right|_{\Omega_{e}} \in H_{\text {loc }}^{\gamma}\left(\Omega_{e}\right)$. By the pointwise multiplier property of $\chi_{\Omega}$, we have $\chi_{\Omega} u \in H^{\alpha}\left(\mathbb{R}^{n}\right)$ for some $\alpha \in\left(-\frac{1}{2}, \frac{1}{2}\right)$ and $(-\Delta)^{s}\left(\chi_{\Omega} u\right) \in H^{\alpha-2 s}\left(\mathbb{R}^{n}\right)$. However, if $\varphi, \psi \in C_{c}^{\infty}\left(\mathbb{R}^{n}\right)$ satisfy $\varphi=1$ near $\bar{\Omega}$ and $\psi=1$ near supp $(\varphi)$, then for any $r, t \in \mathbb{R}$ one has

$$
(1-\psi)(-\Delta)^{s} \varphi: H^{-r}\left(\mathbb{R}^{n}\right) \rightarrow H^{t}\left(\mathbb{R}^{n}\right)
$$

by the pseudolocal property of Fourier multipliers. Thus one also has $\left.(-\Delta)^{s}\left(\chi_{\Omega} u\right)\right|_{\Omega_{e}} \in H_{\mathrm{loc}}^{t}\left(\Omega_{e}\right)$ for any $t$, and $\mathcal{N}_{s}$ is well-defined and maps $H^{\gamma}\left(\mathbb{R}^{n}\right)$ to $H_{\text {loc }}^{\gamma}\left(\Omega_{e}\right)$ for $\gamma>-\frac{1}{2}$. 
Moreover, if $u \in L^{2}\left(\mathbb{R}^{n}\right)$ and if $\varphi_{j} \in C_{c}^{\infty}(\Omega)$ satisfy $\varphi_{j} \rightarrow \chi_{\Omega} u$ in $L^{2}\left(\mathbb{R}^{n}\right)$, then the pseudolocal property implies

$$
\left.\left.(-\Delta)^{s}\left(\varphi_{j}\right)\right|_{\Omega_{e}} \rightarrow(-\Delta)^{s}\left(\chi_{\Omega} u\right)\right|_{\Omega_{e}} \text { in } L_{\mathrm{loc}}^{2}\left(\Omega_{e}\right)
$$

After extracting a subsequence (using the diagonal argument), one has convergence a.e. in $\Omega_{e}$. Thus the pointwise expression (A-2) for a.e. $x \in \Omega_{e}$ follows from the standard formula

$$
(-\Delta)^{s} \varphi(x)=c_{n, s} \int_{\mathbb{R}^{n}} \frac{\varphi(x)-\varphi(y)}{|x-y|^{n+2 s}} d y, \quad \varphi \in C_{c}^{\infty}(\Omega), x \in \Omega_{e} .
$$

Let us prove the formula for $\Lambda_{q}$. If $f \in H^{s+\beta}\left(\Omega_{e}\right)$, then $f \in H^{\alpha}\left(\Omega_{e}\right)$ for some $\alpha \in\left(-\frac{1}{2}, \frac{1}{2}\right)$ and hence $E_{0} f, u_{f} \in H^{\alpha}\left(\mathbb{R}^{n}\right)$. Recall also that $\chi_{\Omega}$ and $1-\chi_{\Omega}$ are pointwise multipliers on $H^{\alpha}\left(\mathbb{R}^{n}\right)$. Then

$$
\begin{aligned}
\Lambda_{q} f & =\left.(-\Delta)^{s} u_{f}\right|_{\Omega_{e}}=\left.(-\Delta)^{s}\left(\chi_{\Omega} u_{f}\right)\right|_{\Omega_{e}}+\left.(-\Delta)^{s}\left(\left(1-\chi_{\Omega}\right) u_{f}\right)\right|_{\Omega_{e}} \\
& =\mathcal{N}_{s} u_{f}-m f+\left.(-\Delta)^{s}\left(E_{0} f\right)\right|_{\Omega_{e}} .
\end{aligned}
$$

Nonlocal diffusion. Finally, we will give a heuristic interpretation of the quantity $\Lambda_{q} f(x)$ in terms of nonlocal diffusions [Andreu-Vaillo et al. 2010]. This discussion is mostly for illustrative purposes, so we will not give precise arguments and will restrict to the case $q=0$.

We begin with a macroscopic description of nonlocal diffusion in $\mathbb{R}^{n}$. Suppose that $u(x, t)$ describes the density of particles at a point $x \in \mathbb{R}^{n}$ at time $t$. Given an initial density $u_{0}(x)$, we assume that $u(x, t)$ is obtained as a solution of the nonlocal diffusion equation

$$
\left\{\begin{aligned}
\partial_{t} u+(-\Delta)^{s} u & =0 \quad \text { in } \mathbb{R}^{n} \times\{t>0\} \\
\left.u\right|_{t=0} & =u_{0} .
\end{aligned}\right.
$$

Taking Fourier transforms in $x$, the solution at time $t$ is given by

$$
u(t, x)=\left(p_{t} * u_{0}\right)(x),
$$

where $p_{t}(x)=\mathscr{F}^{-1}\left\{e^{-t|\xi|^{2 s}}\right\}$ is the probability density function of the Lévy process $X_{t}$ with infinitesimal generator $-(-\Delta)^{s}$. If $s=1$, then $p_{t}$ is a Gaussian, but for $0<s<1$ it is a heavy-tailed distribution with $p_{t}(x) \sim|x|^{-n-2 s}$ for large $|x|$ (for $s=\frac{1}{2}, p_{t}(x)=c_{n} t\left(t^{2}+|x|^{2}\right)^{-(n+1) / 2}$ ). The Lévy process $X_{t}$ also gives a microscopic description of $u(x, t)$ : it is obtained as the expected value

$$
u(x, t)=\mathbb{E}_{x}\left[u_{0}\left(X_{t}\right)\right],
$$

which expresses how many Lévy particles from the initial distribution $u_{0}$ have jumped to $x$ at time $t$. See [Applebaum 2004; Chen et al. 2010] for Lévy processes.

Let now $\Omega \subset \mathbb{R}^{n}$ be a bounded open set. We consider the following Dirichlet problem for nonlocal diffusion: given $u_{0} \in H_{\bar{\Omega}}^{s}$, find $u$ so that

$$
\left\{\begin{aligned}
\partial_{t} u+(-\Delta)^{s} u & =0 \quad \text { in } \Omega \times\{t>0\}, \\
\left.u\right|_{\Omega_{e} \times\{t>0\}} & =0, \\
\left.u\right|_{\mathbb{R}^{n} \times\{t=0\}} & =u_{0} .
\end{aligned}\right.
$$


The solution is easily obtained in the form

$$
u(x, t)=\sum_{j=1}^{\infty} e^{-\lambda_{j} t} c_{j} \rho_{j}(x),
$$

where $u_{0}=\sum_{j=1}^{\infty} c_{j} \rho_{j}$ and $\left\{\rho_{j}\right\}_{j=1}^{\infty} \subset H_{\bar{\Omega}}^{s}$ is an orthonormal basis of $L_{\bar{\Omega}}^{2}$ consisting of eigenfunctions for $(-\Delta)^{s}$ with eigenvalues $\lambda_{j}$, so that $(-\Delta)^{s} \rho_{j}=\lambda_{j} \rho_{j}$ in $\Omega,\left.\rho_{j}\right|_{\Omega_{e}}=0$, and $0<\lambda_{1} \leq \lambda_{2} \leq \cdots \rightarrow \infty$. The probabilistic interpretation is that we are looking at Lévy particles in $\Omega$ that are terminated when they reach the exterior. One has

$$
u(x, t)=\mathbb{E}_{x}\left[u_{0}\left(X_{t}\right) 1_{\{t<\tau\}}\right],
$$

where $\tau$ is the time when the Lévy process exits $\Omega$.

By the Duhamel principle and a standard reduction to homogeneous Dirichlet values, given any $f \in H^{s}\left(\Omega_{e}\right)$ and any $e_{f} \in H^{s}\left(\mathbb{R}^{n}\right)$ with $\left.e_{f}\right|_{\Omega_{e}}=f$, we can also solve the equation

$$
\left\{\begin{aligned}
\partial_{t} v+(-\Delta)^{s} v=0 & & \text { in } \Omega \times\{t>0\} \\
\left.v(\cdot, t)\right|_{\Omega_{e}}=f & & \text { for } t>0
\end{aligned}\right.
$$

with initial value $\left.v\right|_{\mathbb{R}^{n} \times\{t=0\}}=e_{f}$. Another solution of (A-6) is given by $v_{s}(x, t)=u_{f}(x)$, if $u_{f} \in H^{s}\left(\mathbb{R}^{n}\right)$ solves $(-\Delta)^{s} u=0$ in $\Omega$ with $\left.u\right|_{\Omega_{e}}=f$. The function $u_{f}$ is the unique steady state of (A-6), since $v-v_{s}$ solves (A-4) for some $u_{0}$, and (A-5) implies

$$
\left\|v(\cdot, t)-u_{f}\right\|_{H^{s}} \rightarrow 0 \quad \text { as } t \rightarrow \infty .
$$

Now, given $f \in H^{s}\left(\Omega_{e}\right)$ and the solution $u_{f}$ of the Dirichlet problem, we may consider two nonlocal diffusions with initial value $u_{f}$ :

- the free diffusion (A-3) in $\mathbb{R}^{n}$ with solution $u(x, t)$,

- the diffusion (A-6) whose exterior value is fixed to be $f$.

If $t$ is small and $x \in \Omega_{e}$, then $u(x, t)$ formally satisfies

$$
\begin{aligned}
u(x, t) & =u(x, 0)+\partial_{t} u(x, 0) t+O\left(t^{2}\right)=f(x)-(-\Delta)^{s} u(x, 0) t+O\left(t^{2}\right) \\
& =f(x)-\left(\Lambda_{0} f\right)(x) t+O\left(t^{2}\right)
\end{aligned}
$$

by Lemma A.1. Thus the DN map may be interpreted as follows:

- $-\Lambda_{0} f(x)$ is the (infinitesimal) amount of particles migrating to $x$ in the free diffusion that starts from the steady state $u_{f}$.

- $\Lambda_{0} f(x)$ is the (infinitesimal) cost required to maintain the exterior value $f$ at $x$ in the steady state nonlocal diffusion.

Similar remarks apply to $\Lambda_{q}$ at least if $q \geq 0$. We refer to [Chen et al. 2006] for some facts on the related stochastic processes, and to [Piiroinen and Simon 2017] for stochastic interpretations of the usual Calderón problem. 


\section{Acknowledgements}

Salo was partly supported by the Academy of Finland (Centre of Excellence in Inverse Modelling and Imaging, grant numbers 284715 and 309963) and by the European Research Council under FP7/2007-2013 (ERC StG 307023) and Horizon 2020 (ERC CoG 770924). Uhlmann was partly supported by the NSF, a Si-Yuan Professorship at IAS, HKUST, and a FiDiPro at the University of Helsinki. The authors thank Gerd Grubb for several remarks that considerably improved Section 6, and Zhen-Qing Chen for helpful discussions.

\section{References}

[Andreu-Vaillo et al. 2010] F. Andreu-Vaillo, J. M. Mazón, J. D. Rossi, and J. J. Toledo-Melero, Nonlocal diffusion problems, Math. Surveys and Monographs 165, Amer. Math. Soc., Providence, RI, 2010. MR Zbl

[Applebaum 2004] D. Applebaum, Lévy processes and stochastic calculus, Cambridge Studies in Adv. Math. 93, Cambridge Univ. Press, 2004. MR Zbl

[Blåsten et al. 2015] E. Blåsten, O. Y. Imanuvilov, and M. Yamamoto, "Stability and uniqueness for a two-dimensional inverse boundary value problem for less regular potentials", Inverse Probl. Imaging 9:3 (2015), 709-723. MR Zbl

[Bucur and Valdinoci 2016] C. Bucur and E. Valdinoci, Nonlocal diffusion and applications, Lect. Notes Unione Mat. Ital. 20, Springer, 2016. MR Zbl

[Bukhgeim 2008] A. L. Bukhgeim, "Recovering a potential from Cauchy data in the two-dimensional case", J. Inverse Ill-Posed Probl. 16:1 (2008), 19-33. MR Zbl

[Cabré and Sire 2014] X. Cabré and Y. Sire, "Nonlinear equations for fractional Laplacians, I: Regularity, maximum principles, and Hamiltonian estimates", Ann. Inst. H. Poincaré Anal. Non Linéaire 31:1 (2014), 23-53. MR Zbl

[Caffarelli and Silvestre 2007] L. Caffarelli and L. Silvestre, "An extension problem related to the fractional Laplacian", Comm. Partial Differential Equations 32:7-9 (2007), 1245-1260. MR Zbl

[Calderón 1980] A.-P. Calderón, "On an inverse boundary value problem", pp. 65-73 in Seminar on numerical analysis and its applications to continuum physics (Rio de Janeiro, 1980), edited by W. H. Meyer and M. A. Raupp, Soc. Brasil. Mat., Rio de Janeiro, 1980. MR

[Cao et al. 2019] X. Cao, Y.-H. Lin, and H. Liu, "Simultaneously recovering potentials and embedded obstacles for anisotropic fractional Schrödinger operators”, Inverse Probl. Imaging 13:1 (2019), 197-210. MR Zbl

[Chanillo 1990] S. Chanillo, "A problem in electrical prospection and a $n$-dimensional Borg-Levinson theorem”, Proc. Amer. Math. Soc. 108:3 (1990), 761-767. MR Zbl

[Chen et al. 2006] Z.-Q. Chen, M. Fukushima, and J. Ying, "Traces of symmetric Markov processes and their characterizations", Ann. Probab. 34:3 (2006), 1052-1102. MR Zbl

[Chen et al. 2010] Z.-Q. Chen, P. Kim, and R. Song, "Heat kernel estimates for the Dirichlet fractional Laplacian", J. Eur. Math. Soc. 12:5 (2010), 1307-1329. MR Zbl

[Cheng et al. 2009] J. Cheng, J. Nakagawa, M. Yamamoto, and T. Yamazaki, "Uniqueness in an inverse problem for a one-dimensional fractional diffusion equation", Inverse Problems 25:11 (2009), art. id. 115002. MR Zbl

[Daudé et al. 2019a] T. Daudé, N. Kamran, and F. Nicoleau, "Non-uniqueness results for the anisotropic Calderón problem with data measured on disjoint sets", Ann. Inst. Fourier (Grenoble) 69:1 (2019), 119-170. MR Zbl

[Daudé et al. 2019b] T. Daudé, N. Kamran, and F. Nicoleau, "On the hidden mechanism behind non-uniqueness for the anisotropic Calderón problem with data on disjoint sets”, Ann. Henri Poincaré 20:3 (2019), 859-887. MR Zbl

[Dipierro et al. 2017a] S. Dipierro, X. Ros-Oton, and E. Valdinoci, "Nonlocal problems with Neumann boundary conditions", Rev. Mat. Iberoam. 33:2 (2017), 377-416. MR Zbl

[Dipierro et al. 2017b] S. Dipierro, O. Savin, and E. Valdinoci, "All functions are locally s-harmonic up to a small error", J. Eur. Math. Soc. 19:4 (2017), 957-966. MR Zbl 
[Dipierro et al. 2019] S. Dipierro, O. Savin, and E. Valdinoci, "Local approximation of arbitrary functions by solutions of nonlocal equations", J. Geom. Anal. 29:2 (2019), 1428-1455. MR Zbl

[Fall and Felli 2014] M. M. Fall and V. Felli, "Unique continuation property and local asymptotics of solutions to fractional elliptic equations”, Comm. Partial Differential Equations 39:2 (2014), 354-397. MR Zbl

[Felsinger et al. 2015] M. Felsinger, M. Kassmann, and P. Voigt, “The Dirichlet problem for nonlocal operators”, Math. Z. 279:3-4 (2015), 779-809. MR Zbl

[Ghosh et al. 2017] T. Ghosh, Y.-H. Lin, and J. Xiao, "The Calderón problem for variable coefficients nonlocal elliptic operators", Comm. Partial Differential Equations 42:12 (2017), 1923-1961. MR Zbl

[Ghosh et al. 2018] T. Ghosh, A. Rüland, M. Salo, and G. Uhlmann, "Uniqueness and reconstruction for the fractional Calderón problem with a single measurement", preprint, 2018. arXiv

[Grubb 2014] G. Grubb, "Local and nonlocal boundary conditions for $\mu$-transmission and fractional elliptic pseudodifferential operators", Anal. PDE 7:7 (2014), 1649-1682. MR Zbl

[Grubb 2015] G. Grubb, "Fractional Laplacians on domains, a development of Hörmander's theory of $\mu$-transmission pseudodifferential operators", Adv. Math. 268 (2015), 478-528. MR Zbl

[Grubb 2016] G. Grubb, "Regularity of spectral fractional Dirichlet and Neumann problems", Math. Nachr. 289:7 (2016), 831-844. MR Zbl

[Harrach and Lin 2017] B. Harrach and Y.-H. Lin, "Monotonicity-based inversion of the fractional Schrödinger equation, I: Positive potentials", preprint, 2017. arXiv

[Hoh and Jacob 1996] W. Hoh and N. Jacob, "On the Dirichlet problem for pseudodifferential operators generating Feller semigroups", J. Funct. Anal. 137:1 (1996), 19-48. MR Zbl

[Hörmander 1965] L. Hörmander, "Boundary problems for classical pseudo-differential operators", unpublished lecture notes, Inst. Adv. Study, 1965.

[Hörmander 1983] L. Hörmander, The analysis of linear partial differential operators, I: Distribution theory and Fourier analysis, Grundlehren der Math. Wissenschaften 256, Springer, 1983. MR Zbl

[Imanuvilov and Yamamoto 2013] O. Y. Imanuvilov and M. Yamamoto, "Uniqueness for inverse boundary value problems by Dirichlet-to-Neumann map on subboundaries", Milan J. Math. 81:2 (2013), 187-258. MR Zbl

[Imanuvilov et al. 2010] O. Y. Imanuvilov, G. Uhlmann, and M. Yamamoto, "The Calderón problem with partial data in two dimensions", J. Amer. Math. Soc. 23:3 (2010), 655-691. MR Zbl

[Imanuvilov et al. 2011] O. Y. Imanuvilov, G. Uhlmann, and M. Yamamoto, "Inverse boundary value problem by measuring Dirichlet data and Neumann data on disjoint sets", Inverse Problems 27:8 (2011), art. id. 085007. MR Zbl

[Isakov 1990] V. Isakov, Inverse source problems, Math. Surveys and Monographs 34, Amer. Math. Soc., Providence, RI, 1990. MR Zbl

[Isakov 2007] V. Isakov, "On uniqueness in the inverse conductivity problem with local data”, Inverse Probl. Imaging 1:1 (2007), 95-105. MR Zbl

[Jin and Rundell 2015] B. Jin and W. Rundell, "A tutorial on inverse problems for anomalous diffusion processes", Inverse Problems 31:3 (2015), art. id. 035003. MR Zbl

[Kenig and Salo 2013] C. Kenig and M. Salo, "The Calderón problem with partial data on manifolds and applications", Anal. PDE 6:8 (2013), 2003-2048. MR Zbl

[Kenig and Salo 2014] C. Kenig and M. Salo, "Recent progress in the Calderón problem with partial data", pp. 193-222 in Inverse problems and applications (Irvine, CA, 2012/Hangzhou, 2012), edited by P. Stefanov et al., Contemp. Math. 615, Amer. Math. Soc., Providence, RI, 2014. MR Zbl

[Kenig et al. 2007] C. E. Kenig, J. Sjöstrand, and G. Uhlmann, "The Calderón problem with partial data", Ann. of Math. (2) 165:2 (2007), 567-591. MR Zbl

[Kwaśnicki 2017] M. Kwaśnicki, “Ten equivalent definitions of the fractional Laplace operator”, Fract. Calc. Appl. Anal. 20:1 (2017), 7-51. MR Zbl

[Lai and Lin 2019] R.-Y. Lai and Y.-H. Lin, "Global uniqueness for the fractional semilinear Schrödinger equation", Proc. Amer. Math. Soc. 147:3 (2019), 1189-1199. MR Zbl 
[Lax 1956] P. D. Lax, "A stability theorem for solutions of abstract differential equations, and its application to the study of the local behavior of solutions of elliptic equations", Comm. Pure Appl. Math. 9 (1956), 747-766. MR Zbl

[Malgrange 1956] B. Malgrange, "Existence et approximation des solutions des équations aux dérivées partielles et des équations de convolution", Ann. Inst. Fourier (Grenoble) 6 (1956), 271-355. MR Zbl

[McLean 2000] W. McLean, Strongly elliptic systems and boundary integral equations, Cambridge Univ. Press, 2000. MR Zbl

[Nachman 1992] A. I. Nachman, "Inverse scattering at fixed energy", pp. 434-441 in Proceedings of the Tenth Congress on Mathematical Physics (Leipzig, 1991), edited by K. Schmüdgen, Springer, 1992. MR Zbl

[Piiroinen and Simon 2017] P. Piiroinen and M. Simon, "Probabilistic interpretation of the Calderón problem", Inverse Probl. Imaging 11:3 (2017), 553-575. MR Zbl

[Riesz 1938] M. Riesz, "Intégrales de Riemann-Liouville et potentiels”, Acta Litt. Sci. Szeged 9 (1938), 1-42. Zbl

[Ros-Oton 2016] X. Ros-Oton, "Nonlocal elliptic equations in bounded domains: a survey", Publ. Mat. 60:1 (2016), 3-26. MR $\mathrm{Zbl}$

[Rüland 2015] A. Rüland, "Unique continuation for fractional Schrödinger equations with rough potentials", Comm. Partial Differential Equations 40:1 (2015), 77-114. MR Zbl

[Rüland and Salo 2018] A. Rüland and M. Salo, "Exponential instability in the fractional Calderón problem", Inverse Problems 34:4 (2018), art. id. 045003. MR Zbl

[Rüland and Salo 2019a] A. Rüland and M. Salo, “The fractional Calderón problem: low regularity and stability", Nonlinear Anal. (online publication May 2019).

[Rüland and Salo 2019b] A. Rüland and M. Salo, "Quantitative Runge approximation and inverse problems", Int. Math. Res. Not. 2019:20 (2019), 6216-6234. Zbl

[Rüland and Salo 2020] A. Rüland and M. Salo, "Quantitative approximation properties for the fractional heat equation", Math. Control Related Fields 10:1 (2020), 1-26.

[Sakamoto and Yamamoto 2011] K. Sakamoto and M. Yamamoto, "Initial value/boundary value problems for fractional diffusion-wave equations and applications to some inverse problems”, J. Math. Anal. Appl. 382:1 (2011), 426-447. MR Zbl

[Salo 2017] M. Salo, “The fractional Calderón problem”, J. Éq. Dérivées Partielles 2017 (2017), art. id. 7.

[Silvestre 2007] L. Silvestre, "Regularity of the obstacle problem for a fractional power of the Laplace operator", Comm. Pure Appl. Math. 60:1 (2007), 67-112. MR Zbl

[Sylvester and Uhlmann 1987] J. Sylvester and G. Uhlmann, "A global uniqueness theorem for an inverse boundary value problem", Ann. of Math. (2) 125:1 (1987), 153-169. MR Zbl

[Taylor 1996] M. E. Taylor, Partial differential equations, III: Nonlinear equations, Appl. Math. Sci. 117, Springer, 1996. Zbl

[Triebel 2002] H. Triebel, "Function spaces in Lipschitz domains and on Lipschitz manifolds: characteristic functions as pointwise multipliers”, Rev. Mat. Complut. 15:2 (2002), 475-524. MR Zbl

[Uhlmann 2014] G. Uhlmann, "Inverse problems: seeing the unseen”, Bull. Math. Sci. 4:2 (2014), 209-279. MR Zbl

[Vishik and Eskin 1965] M. I. Vishik and G. I. Eskin, "Equations in convolutions in a bounded region", Uspehi Mat. Nauk 20:3(123) (1965), 89-152. In Russian; translated in Russian Math. Surv. 20:3 (1965), 85-151. MR Zbl

[Yu 2017] H. Yu, "Unique continuation for fractional orders of elliptic equations", Ann. PDE 3:2 (2017), art. id. 16. MR Zbl

Received 9 Jul 2018. Revised 8 Nov 2018. Accepted 23 Feb 2019.

TuHIN GHOSH: imaginetuhin@gmail.com

Jockey Club Institute for Advanced Study, Hong Kong University of Science and Technology, Kowloon, Hong Kong

MikKo SALO: mikko.j.salo@jyu.fi

Department of Mathematics and Statistics, University of Jyväskylä, Jyväskylä, Finland

GUNTHER UHLMANN: gunther@math.washington.edu

Department of Mathematics, University of Washington, Seattle, WA, United States

and

Jockey Club Institute for Advanced Study, Hong Kong University of Science and Technology, Kowloon, Hong Kong 


\title{
Analysis \& PDE
}

msp.org/apde

\section{EDITORS}

\author{
EDITOR-IN-CHIEF \\ Patrick Gérard \\ patrick.gerard@math.u-psud.fr \\ Université Paris Sud XI \\ Orsay, France
}

BOARD OF EDITORS

$\begin{array}{cl}\text { Massimiliano Berti } & \begin{array}{l}\text { Scuola Intern. Sup. di Studi Avanzati, Italy } \\ \text { berti@ @issa.it }\end{array} \\ \text { Michael Christ } & \begin{array}{l}\text { University of California, Berkeley, USA } \\ \text { mchrist@ math.berkeley.edu }\end{array} \\ \text { Charles Fefferman } & \begin{array}{l}\text { Princeton University, USA } \\ \text { cf@math.princeton.edu }\end{array} \\ \text { Ursula Hamenstaedt } & \begin{array}{l}\text { Universität Bonn, Germany } \\ \text { ursula@math.uni-bonn.de }\end{array} \\ \text { Vadim Kaloshin } & \begin{array}{l}\text { University of Maryland, USA } \\ \text { vadim.kaloshin@ gmail.com }\end{array} \\ \text { Herbert Koch } & \begin{array}{l}\text { Universität Bonn, Germany } \\ \text { koch@math.uni-bonn.de }\end{array} \\ \text { Izabella Laba } & \begin{array}{l}\text { University of British Columbia, Canada } \\ \text { ilaba@math.ubc.ca }\end{array} \\ \text { Richard B. Melrose } & \begin{array}{l}\text { Massachussets Inst. of Tech., USA } \\ \text { rbm@math.mit.edu }\end{array} \\ & \text { Université de Cergy-Pontoise, France } \\ \text { Frank Merle } & \begin{array}{l}\text { Frank.Merle@u-cergy.fr } \\ \text { Johns Hopkins University, USA } \\ \text { minicozz@math.jhu.edu } \\ \text { Cambridge University, UK } \\ \text { c.mouhot@dpmms.cam.ac.uk } \\ \text { Universität Bonn, Germany } \\ \text { mueller@math.uni-bonn.de }\end{array} \\ \text { William Minicozzi II } & \end{array}$

Gilles Pisier Texas A\&M University, and Paris 6 pisier@math.tamu.edu

Tristan Rivière ETH, Switzerland riviere@math.ethz.ch

Igor Rodnianski Princeton University, USA irod@math.princeton.edu

Yum-Tong Siu Harvard University, USA siu@math.harvard.edu

Terence Tao University of California, Los Angeles, USA tao@math.ucla.edu

Michael E. Taylor Univ. of North Carolina, Chapel Hill, USA met@math.unc.edu

Gunther Uhlmann University of Washington, USA gunther@math.washington.edu

András Vasy Stanford University, USA andras@math.stanford.edu

Dan Virgil Voiculescu University of California, Berkeley, USA dvv@math.berkeley.edu

Steven Zelditch Northwestern University, USA zelditch@math.northwestern.edu

Maciej Zworski University of California, Berkeley, USA zworski@math.berkeley.edu

PRODUCTION

production@msp.org

Silvio Levy, Scientific Editor

See inside back cover or msp.org/apde for submission instructions.

The subscription price for 2020 is US $\$ 340 /$ year for the electronic version, and $\$ 550 /$ year $(+\$ 60$, if shipping outside the US) for print and electronic. Subscriptions, requests for back issues from the last three years and changes of subscriber address should be sent to MSP.

Analysis \& PDE (ISSN 1948-206X electronic, 2157-5045 printed) at Mathematical Sciences Publishers, 798 Evans Hall \#3840, c/o University of California, Berkeley, CA 94720-3840, is published continuously online. Periodical rate postage paid at Berkeley, CA 94704, and additional mailing offices.

APDE peer review and production are managed by EditFlow ${ }^{\circledR}$ from MSP.

\section{PUBLISHED BY}

\section{mathematical sciences publishers}

nonprofit scientific publishing

http://msp.org/

(C) 2020 Mathematical Sciences Publishers 


\section{ANALYSIS \& PDE}

\section{Volume $13 \quad$ No. $2 \quad 2020$}

Regularity estimates for elliptic nonlocal operators

BARTŁOMIEJ DYDA and MORITZ KASSMANN

On solvability and ill-posedness of the compressible Euler system subject to stochastic forces

DOMINIC BREIT, EDUARD FEIREISL and MARTINA HOFMANOVÁ

Variable coefficient Wolff-type inequalities and sharp local smoothing estimates for wave equations on manifolds

David Beltran, Jonathan Hickman and Christopher D. SOgGe

On the Hölder continuous subsolution problem for the complex Monge-Ampère equation, II NGOC CUONG NGUYEN

The Calderón problem for the fractional Schrödinger equation

Tuhin GHosh, MikKo SALO and Gunther UhlmanN

Sharp Strichartz inequalities for fractional and higher-order Schrödinger equations

GianMaRco Brocchi, Diogo OliveIRA E Silva and RENÉ QUILODRÁN

371

403

A bootstrapping approach to jump inequalities and their applications

MARIUSZ MireK, Elias M. STEIN and PAVEL ZORIN-KRANICH

On the trace operator for functions of bounded $A$-variation

DOMINIC BREIT, LARS DIENING and FRANZ GMEINEDER

Optimal constants for a nonlocal approximation of Sobolev norms and total variation

Clara Antonucci, Massimo Gobbino, Matteo Migliorini and Nicola PICENNI 